Invited review

\title{
Stress-related regulation of the kynurenine pathway: Relevance to neuropsychiatric and degenerative disorders
}

\author{
Katherine O'Farrell a , Andrew Harkin a, b, * \\ ${ }^{a}$ Neuropsychopharmacology Research Group, School of Pharmacy and Pharmaceutical Sciences \& Trinity College Institute of Neuroscience, Trinity College \\ Dublin, Ireland \\ ${ }^{\mathrm{b}}$ Neuroimmunology Research Group, Department of Physiology, School of Medicine \& Trinity College Institute of Neuroscience, Trinity College Dublin, \\ Ireland
}

\section{A R T I C L E I N F O}

\section{Article history:}

Received 15 October 2015

Received in revised form

2 December 2015

Accepted 8 December 2015

Available online $\mathrm{xxx}$

\section{Keywords:}

Stress

Inflammation

Kynurenine pathway

Neurodegenerative diseases

Psychiatric disorders

\begin{abstract}
A B S T R A C T
The kynurenine pathway (KP), which is activated in times of stress and infection has been implicated in the pathophysiology of neurodegenerative and psychiatric disorders. Activation of this tryptophan metabolising pathway results in the production of neuroactive metabolites which have the potential to interfere with normal neuronal functioning which may contribute to altered neuronal transmission and the emergence of symptoms of these brain disorders. This review investigates the involvement of the KP in a range of neurological disorders, examining recent in vitro, in vivo and clinical discoveries highlights evidence to indicate that the KP is a potential therapeutic target in both neurodegenerative and stressrelated neuropsychiatric disorders. Furthermore, this review identifies gaps in our knowledge with regard to this field which are yet to be examined to lead to a more comprehensive understanding of the role of KP activation in brain health and disease.
\end{abstract}

(C) 2015 Elsevier Ltd. All rights reserved.

\section{Contents}

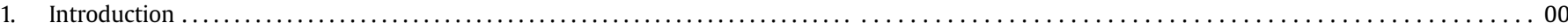

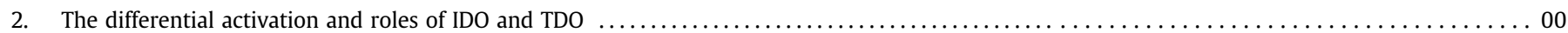

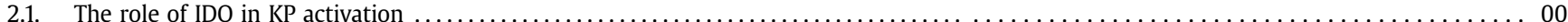

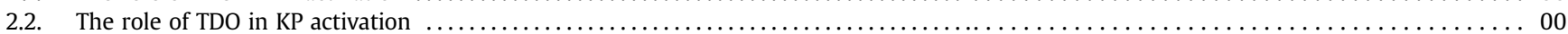

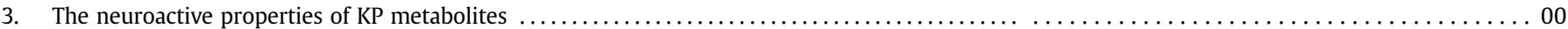

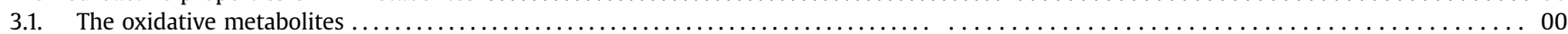

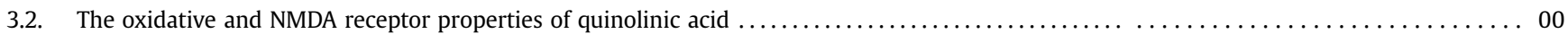

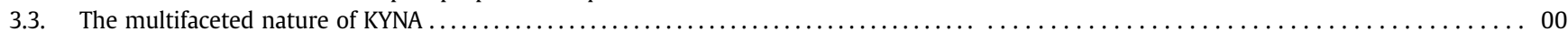

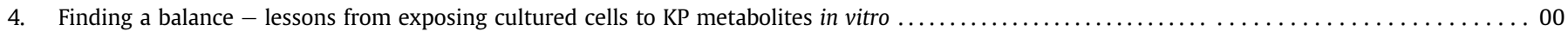

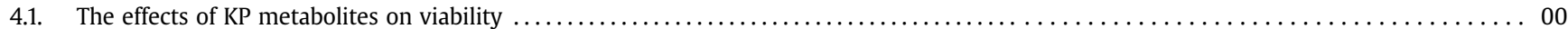

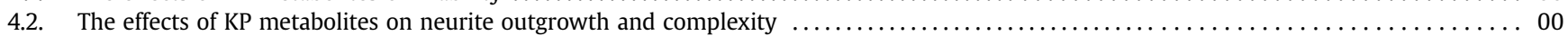

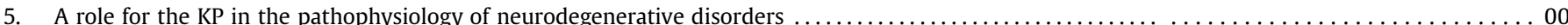

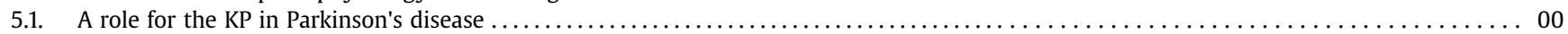

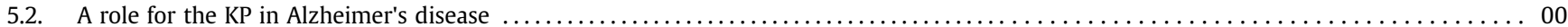

Abbreviations: 3-HAO, 3-hydroxyanthranilic acid 3, 4-dioxygenase; ACMSD, aminocarboxymuconate semialdehyde decarboxylase; AhR, aryl hydrocarbon receptor; AMPA

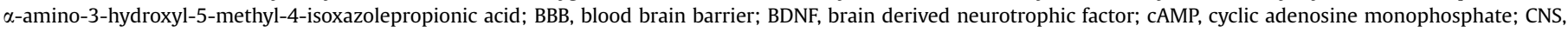

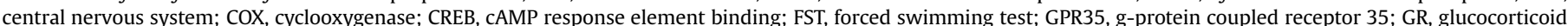

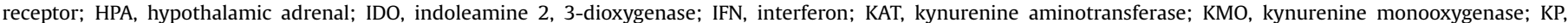

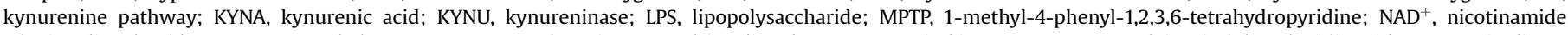

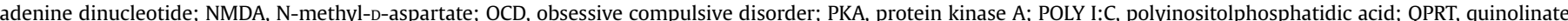

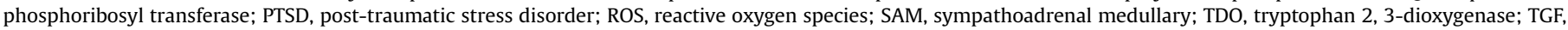
transforming growth factor; TMF, 6,2', $4^{\prime}$-trimethoxyflavone.

* Corresponding author. Trinity College Institute of Neuroscience, Room 4.57, The Lloyd Institute, Trinity College Dublin, Dublin 2, Ireland.

E-mail address: aharkin@tcd.ie (A. Harkin). 


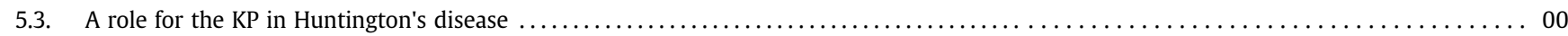

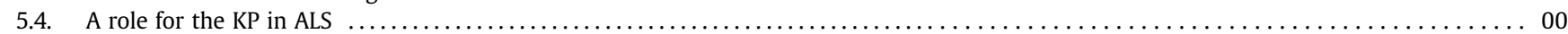

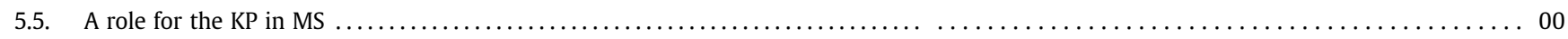

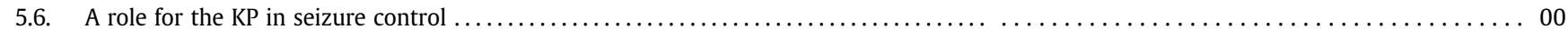

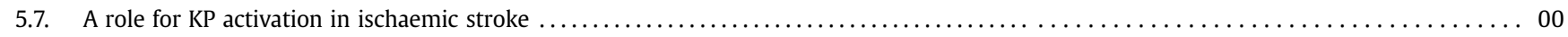

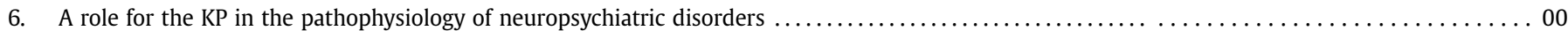

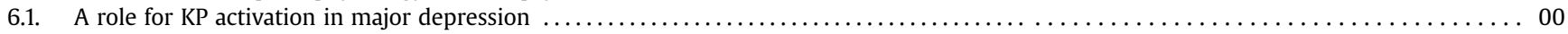

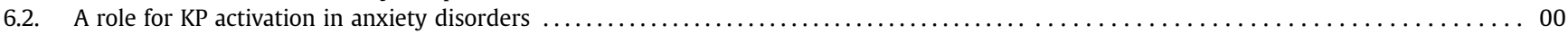

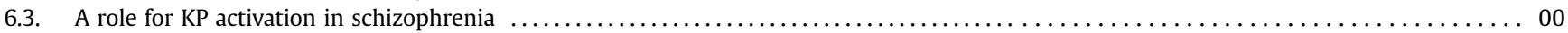

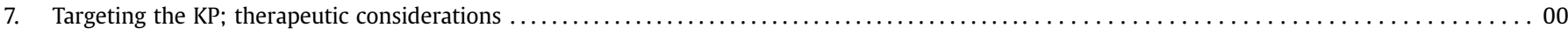

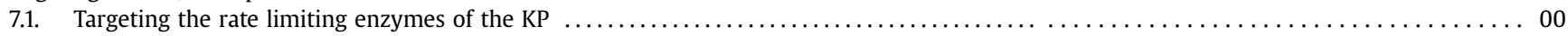

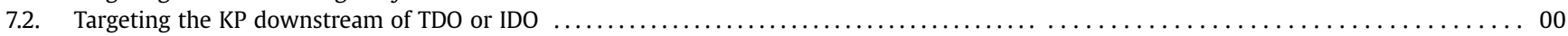

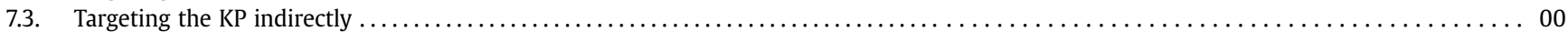

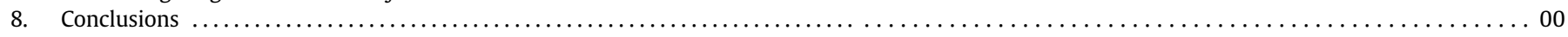

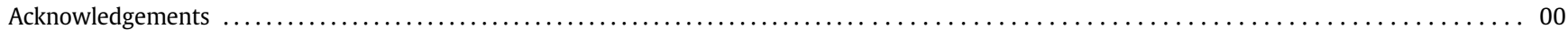

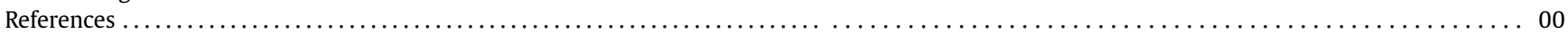

\section{Introduction}

The kynurenine pathway (KP) is a tryptophan metabolism pathway that is induced in times of stress and or immune activation. Initially tryptophan is converted to kynurenine which is subsequently converted into a range of metabolites which have neuromodulatory properties (Fig. 1). The pathway has been implicated in the pathophysiology of multiple central nervous system (CNS) disorders ranging from psychiatric disorders to neurodegenerative diseases (Reus et al., 2015; Bohar et al., 2015; KarakułaJuchnowicz et al., 2015). Its likely contribution to underlying mechanisms associated with CNS disorders indicates that regulation of the KP is of critical importance and may serve as an important target for the future development of treatments for a range of CNS-related illnesses.

\section{The differential activation and roles of IDO and TDO}

Both TDO and IDO have evolved to have similar functions, differing primarily in substrate specificity and tissue and cellular localisation. Most species contain both tryptophan-metabolising enzymes, with gene duplications resulting in certain species having several homologs of these enzymes (Ball et al., 2014). IDO is a monomeric enzyme with a broader substrate specificity than TDO. IDO and TDO have a sequence identity of only $10 \%$, while the recently discovered tryptophan-catabolising enzyme IDO-2 which is encoded by a gene adjacent to that of IDO, shares $43 \%$ sequence identity and structural similarities with IDO (Forouhar et al., 2007). IDO is expressed extra-hepatically in intestinal, lung, placenta and brain tissue (Stone, 1993) with high expression of IDO found in the spleen as a consequence of the accumulation of IDO-expressing immune cells, including dendritic cells and peripheral blood mononuclear cells (Jones et al., 2015; Bronte and Pittet, 2013; Hwu et al., 2000).

\subsection{The role of IDO in KP activation}

Induction of IDO is pivotal in the immune response. IDO activation is associated with the anti-parasitic, anti-fungal, anti-viral and anti-bacterial activities of polymorphonuclear immune cells (Bozza et al., 2005; Kwidzinski and Bechmann, 2007). These effects are achieved through tryptophan depletion and the production of kynurenine, 3-hydroxykynurenine, and 3-hydroxyanthranilic acid in pathogens which are anti-proliferative and increase the apoptotic susceptibility of the cells in which they are produced. Moreover, activation of IDO results in immunosuppression and can lead to immune tolerance (Kwidzinski and Bechmann, 2007) if the aforementioned mechanisms occur in T lymphocytes (Fallarino et al., 2002). The KP metabolites also shift the T cell response towards the development of regulatory $\mathrm{T}$ cells. This occurs as a consequence of a positive feedback cycle which exists between dendritic cells and regulatory T cells, in so much as IDO induction in dendritic cells drives the development of $\mathrm{CD}^{+}{ }^{+} \mathrm{T}$ cells towards the regulatory $\mathrm{T}$ cell phenotype, which further induces IDO in dendritic cells (Hill et al., 2007). In addition, 3-hydroxyanthranilic acid triggers the production of transforming growth factor $\beta$ (TGF $\beta$ ) which further promotes the development of regulatory $\mathrm{T}$ cells, alongside suppressing the development of $\mathrm{Th}_{1}$ cells (Munn, 2011).

In the CNS, IDO is expressed in neurons, macrophages, microglia and astrocytes, but not oligodendrocytes (Lim et al., 2010). Neurons express both IDO and IDO-2 which exhibit a reciprocal relationship with TDO expression, in that induction of IDO results in decreased expression of TDO (Guillemin et al., 2007). IDO-2 is present in different tissues to that of IDO, such as the epididymis, liver and kidneys (Fukunaga et al., 2012; Ball et al., 2007) indicating that it is not functionally redundant. However, the neurological and immune effects of IDO-2 are not fully elucidated and its function in human cells is unknown (Vecsei et al., 2013; Fatokun et al., 2013). Moreover, IDO-2 appears to be much less enzymatically active than IDO as indicated by its lower substrate binding affinity and lower turnover rates (Pantouris et al., 2014).

Activation of the sympathoadrenal medullary (SAM) axis following exposure to stress leads to the release of noradrenaline from sympathetic nerve endings and adrenaline from the adrenal medulla. Catecholamines activate $\beta$-adrenergic receptors expressed on natural killer cells, T cells, B cells and monocytes (Maisel et al., 1989) which results in the expression and release of pro-inflammatory cytokines, specifically interferon- $\gamma$ (IFN $\gamma$ ), IL- $1 \beta$ and IL- 6 which are capable of inducing IDO (Elenkov et al., 2000; see, Kohm and Sanders (2001) for review). Inflammation is also capable of activating the downstream KP enzyme KMO (Connor et al., 2008), which is a flavin adenine dinucleotide dependent monooxygenase enzyme (Alberati-Giani et al., 1997), located in the outer mitochondrial membrane (Erickson et al., 1992). KMO is expressed peripherally in the kidney and liver as well as in the brain where it is primarily found in microglia (Guillemin et al., 2005b), with low expression of it in neurons (Guillemin et al., 2007).

\subsection{The role of TDO in KP activation}

TDO is a homotetrameric enzyme with its expression primarily restricted to the liver in the periphery (Stone, 1993) while it is 


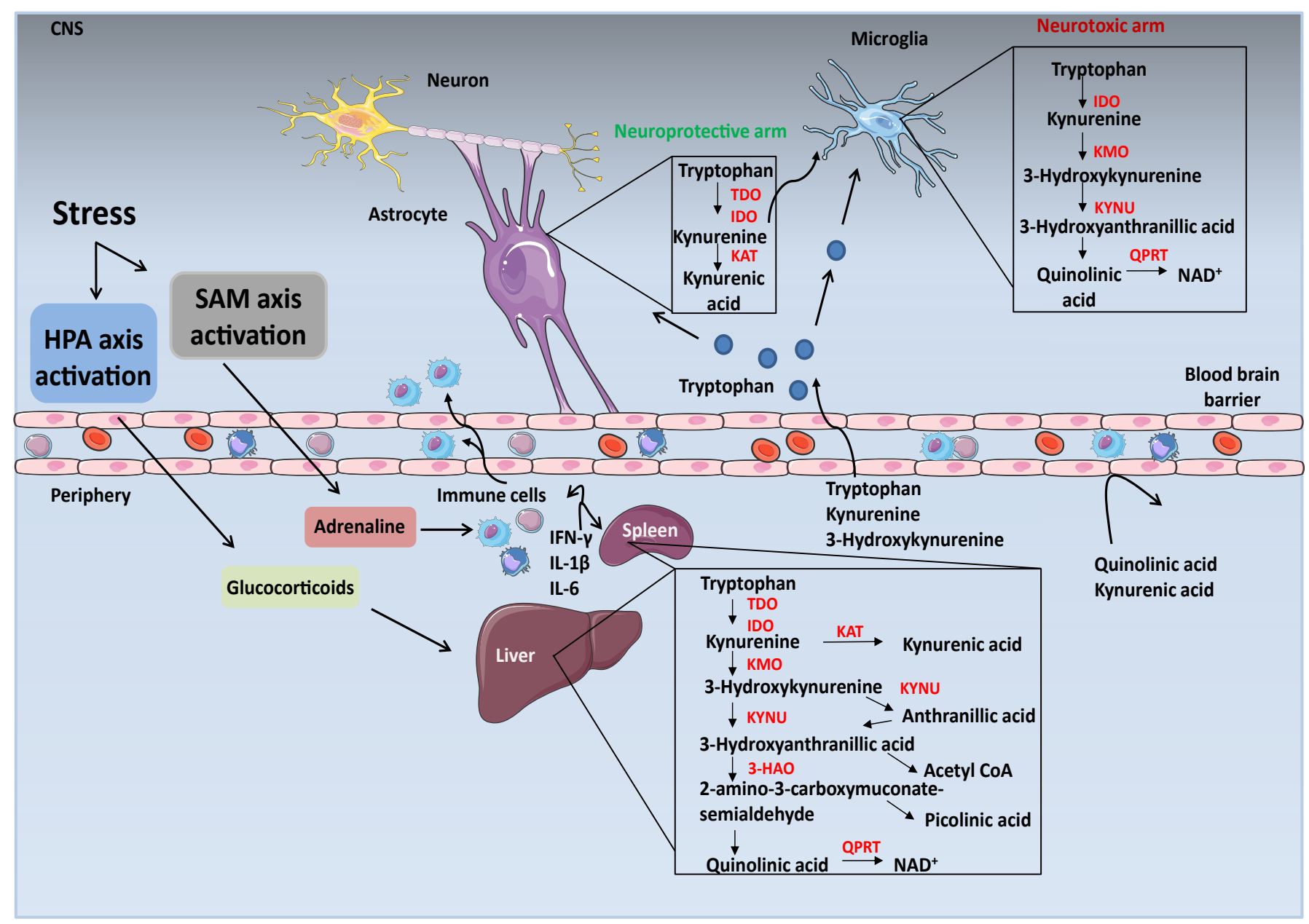

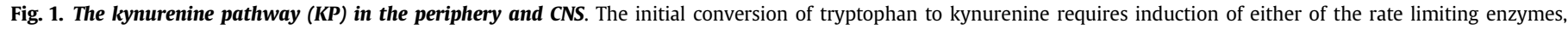

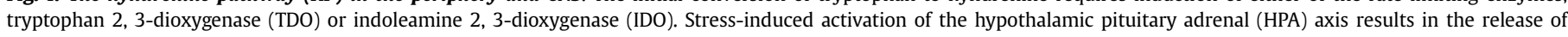

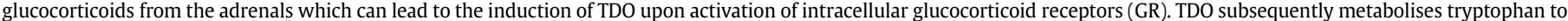

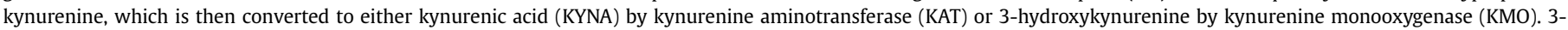

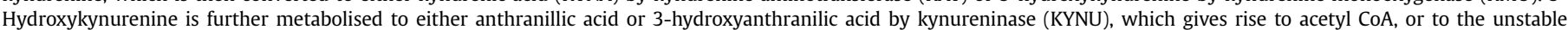

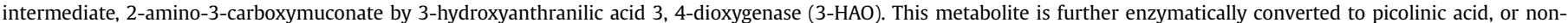

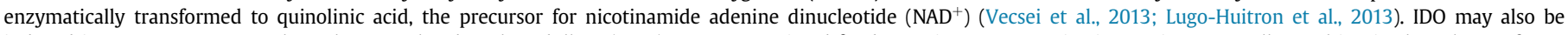

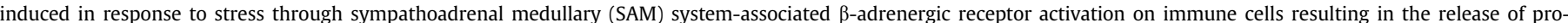

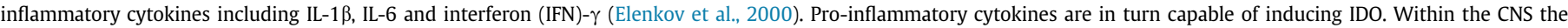

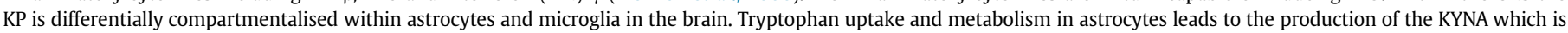

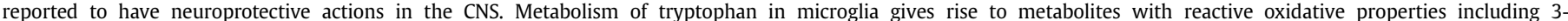

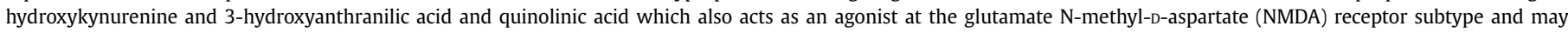

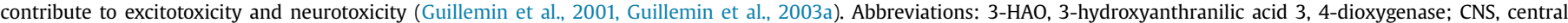

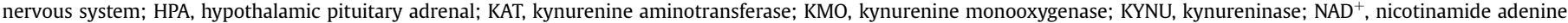
dinucleotide; NMDA, N-methyl-D-aspartate; QPRT, quinolinate phosphoribosyl transferase; SAM, sympathoadrenal medullary.

present in both astrocytes and neurons in the brain (Guillemin et al., 2007; Miller et al., 2004). In fact, there are now three variants of the tdo gene which have been identified in adult mice referred to as tdo variant $1, t d o$ variant 2 , and the classic gene, tdo full-form (Kanai et al., 2009b). Under normal circumstances, TDO activity is regulated by tryptophan's availability with its activity being relatively stable (Myint and Kim, 2014). Regulation of TDO expression occurs chiefly through GR mediated induction. Consequently, stress-related changes in the expression of TDO are primarily influenced by activation of the HPA axis through the action of glucocorticoids (Green and Curzon, 1975).

Considering the nature of factors which influence the expression of IDO and TDO, the relevance of KP induction in times of stress is unclear. It is believed that induction of TDO is a protective mechanism against the unfavourable effects of excess tryptophan which are mediated through the production of indoles, which are capable of disrupting the membrane potential (Kim et al., 2013). In addition, induction of hepatic TDO in times of stress may be important for driving the synthesis of $\mathrm{NAD}^{+}$. This would enable further energy production for use in other immune reactions, while induction of central TDO provides astrocytes with the substrate for the production of KYNA which in turn may have important neuromodulatory actions in response to stress. Continuous generation of $\mathrm{NAD}^{+}$is essential to cell survival (Ying, 2006). For one, it is of utmost importance in the generation of energy (Berger et al., 1986), as well as being involved in the regulation of intracellular calcium and gene expression (Berger et al., 2004; Sauve et al., 2006).

Stimulation of both $\beta$-adrenoceptors and GRs results in receptor downregulation providing a mechanism of control to prevent oversignalling (Asakura et al., 2000; Dong et al., 1988). Although the 
action of glucocorticoids is known to result in downregulation of its receptor in HPA axis tissues, it is not well established if glucocrticoid receptor desensitivity occurs in the liver or immune cells or how TDO/IDO induction are affected by chronic stress or glucocorticoid exposure. Chronic stress has been reported to be accompanied by increased circulating glucocorticoids and resistance to glucocorticoids in both humans and animal models of stress (Miller et al., 2008; Chiba et al., 2012). GR resistance involves the reduced sensitivity of immune cells to glucocorticoids which would otherwise regulate the inflammatory response system (Stark et al., 2001; Marques et al., 2009).

\section{The neuroactive properties of KP metabolites}

\subsection{The oxidative metabolites}

3-Hydroxykynurenine and 3-hydroxyanthranilic acid are KP metabolites which contribute to the generation of free radicals (Forrest et al., 2004) leading to lipid peroxidation and oxidative stress. This is achieved directly through the generation of reactive oxygen species (ROS) such as hydrogen peroxide from these metabolites (Okuda et al., 1996). Moreover, 3-hydroxykynurenine, 3hydroxyanthranilic acid and anthranillic acid can inhibit complexes I, II and IV, I and II, and I-III in the electron transport chain respectively (Schuck et al., 2007). This inhibition may lead to impaired mitochondrial function contributing further to oxidative stress within cells (Maes et al., 2011). Activation of oxidative and nitrosative pathways, and impairments in mitochondrial function are widely reported to be involved in the pathology of neurodegenerative and neuropsychiatric disorders (Glass et al., 2010; Maes, 2011; Federico et al., 2012; Muller, 2013).

\subsection{The oxidative and NMDA receptor properties of quinolinic acid}

Quinolinic acid is a weak but specific competitive agonist at the NMDA receptor containing NR2A and NR2B subunits (Guillemin, 2012). The concentrations of quinolinic acid in the human brain are usually less than $100 \mathrm{nM}$ but these can increase drastically in inflamed tissues (Guillemin et al., 2005a; Guillemin and Brew, 2002; Guidetti and Schwarcz, 2003). In addition to it being directly able to generate ROS (Behan et al., 1999), quinolinic acid can also act through the activation of the NMDA receptor indirectly to drive free radical formation. This can occur through the conversion of xanthine dehydrogenase into xanthine oxidase - an enzyme responsible for the production of ROS, and also through stimulation of nitric oxide synthase (Forrest et al., 2004). Moreover, quinolinic acid stimulates the release of glutamate from neurons (Tavares et al., 2002) and inhibits the uptake of glutamate by astrocytes, enhancing its synaptic availability which in turn may overactivate the NMDA receptor (Tavares et al., 2002). Furthermore, quinolinic acid also impairs postsynaptic elements, causes neuronal degeneration (Kerr et al., 1998), and destabilizes the cytoskeleton (Pierozan et al., 2010). In addition, quinolinic acid triggers proinflammatory responses and apoptosis of neurons and astrocytes (Braidy et al., 2009a), as well as disrupting the blood brain barrier (BBB) (St'astny et al., 1999). Further effects of quinolinic acidinduced cytotoxicity have been discussed elsewhere (LugoHuitron et al., 2013).

\subsection{The multifaceted nature of KYNA}

KYNA is found in the human brain at concentrations between 0.2 and $1.5 \mu \mathrm{M}$ (Moroni et al., 1988). It is produced from kynurenine by the KAT enzymes of which there are 4, KAT I, II, III, IV, all of which are involved to varying degrees in the conversion of kynurenine to
KYNA (see also Fig. 1) (Agudelo et al., 2014; Zinger et al., 2011). Within the brain, KAT II is mostly responsible for the production of KYNA (75\%) (Zinger et al., 2011). KAT II mediated conversion is the primary source for KYNA production in the brain due to the inability of KYNA to cross the BBB (Fukui et al., 1991). KYNA is often referred to as a neuroprotective KP metabolite on account of antagonistic properties at the NMDA receptor (Stone, 1993) which may serve to counteract quinolinic acid-related toxicity (Perkins and Stone, 1982). Specifically KYNA is an antagonist at the glycine recognition and co-agonist site of the NMDA receptor complex (Birch et al., 1988b). Consequently, the ratio of quinolinic acid to KYNA is used as an index to gauge for NMDA receptor-related activation and neurotoxicity (Saito et al., 1993). At higher concentrations KYNA has the capacity to antagonise the NMDA receptor at the glutamate binding site (Birch et al., 1988a), thus KYNA has the potential to interfere with glutamatergic signalling. In addition to its NMDA receptor antagonistic properties, KYNA also blocks other glutamate receptors including kainite and $\alpha$-amino-3-hydroxyl-5methyl-4-isoxazolepropionic acid (AMPA) receptors (Birch et al., 1988a). It is also a non-competitive inhibitor at the $\alpha 7$-nicotinic receptor (Hilmas et al., 2001), an important component of the cholinergic anti-inflammatory pathway that inhibits cytokine activities and glutamate release (Gallowitsch-Puerta and Tracey, 2005; Marchi et al., 2002). The actions of KYNA at the $\alpha 7-$ nicotinic receptor and the glycine binding site of the NMDA receptor occur at more physiologically relevant concentrations (Prescott et al., 2006; Szalardy et al., 2012), while its antagonism at the AMPA receptor or at the NMDA binding site of the NMDA receptor occurs at higher concentrations (Szalardy et al., 2012).

The concentrations of KYNA in the brain have been proposed to be regulated by $\beta$-adrenoceptor stimulation in a cyclic adenosine monophosphate (cAMP)- and protein kinase A (PKA)-dependent manner (Luchowska et al., 2009). Luchowska et al. (2009) demonstrated that stimulation of either rat cortical slices or rat mixed glial cultures with either $\beta$-adrenoceptor agonists or cAMP analogues resulted in increased KYNA concentrations which was blocked by the PKA inhibitor, KT5720. Similarly, the $\beta$-adrenoceptor agonist formoterol resulted in increased KAT I and II activity in mixed glial cultures. Other mechanisms by which the induction of central KAT and increased KYNA production occur are unclear. However, it has been demonstrated that sulphur-containing acidic amino acids, which exert neuroexcitatory actions similar those of glutamate, reduce KYNA production and inhibit KAT I and II activity in the brains of Wistar rats (Kocki et al., 2003).

Despite its reputation as a neuroprotective metabolite, KYNA has been found to be a full agonist at the orphan receptor, gprotein coupled receptor 35 (GPR35) in rodents (Wang et al., 2006). Activation of this receptor results in $G_{\alpha 13}$ and $G_{i / o}$ signalling leading to mobilisation of intracellular calcium and the disruption of synaptic currents (Wang et al., 2006; Jenkins et al., 2011; Divorty et al., 2015). The degree of potency of KYNA at GPR35 is species specific, with it being more potent at rat in comparison with human GPR35 (Wang et al., 2006). Considering that GPR35 is not widely distributed in the brain (Wang et al., 2006), it is more likely that KYNA exerts its action via this receptor in the periphery, primarily in the gastrointestinal tract or immune cells where GPR35 is predominantly expressed (Wang et al., 2006). Furthermore, the plasma concentration of KYNA required to activate GPR35 is not reached under normal circumstances in humans, suggesting that KYNA-driven GPR35 activation may only be relevant in the presence of disease salient stimuli (Divorty et al., 2015). In spite of the potential of KYNA to perturb neuronal signalling through GPR35 activation, further studies are required in order to determine whether this receptor may influence the neuroactive properties of KYNA. 


\section{Finding a balance - lessons from exposing cultured cells to KP metabolites in vitro}

\subsection{The effects of KP metabolites on viability}

3-Hydroxykynurenine, and to a lesser extent 3hydroxyanthranilic acid, at concentrations ranging from 1 to $100 \mu \mathrm{M}$ were reported to be toxic to rat striatal neurons where toxicity was prevented by treatment with the xanthine oxidase inhibitor, allopurinol (Okuda et al., 1996). Furthermore, the same group reported that other KP metabolites tested including quinolinic acid over a similar range of $\mu \mathrm{M}$ concentrations did not affect the viability of these cultured striatal neurons (Okuda et al., 1998). In another report, 3-hydroxykynurenine was capable of inducing cell death in both undifferentiated and differentiated SH-SY5Y and SK-N-SH neuroblastoma cells (Wszelaki and Melzig, 2011). 3Hydroxykynurenine, 3-hydroxyanthranilic acid and quinolinic acid, but not KYNA have been reported to affect the viability of human neuronal cells with lactate dehydrogenase release from cells indicative of neuronal cell death at doses greater than $0.5 \mu \mathrm{M}$ (Braidy et al., 2009b). Similarly this group assessed the viability of human astrocytes following exposure to various KP metabolites, and they found that 3-hydroxykynurenine, 3-hydroxyanthranilic acid and quinolinic acid, but not KYNA reduced astrocytic viability (Braidy et al., 2009a). In a further report, the quinolinic acidinduced reduction in neuronal viability was prevented by the pre-treatment with the NMDA receptor antagonist, MK-801 or the inducible nitric oxide (iNOS) inhibitor, L-NAME. Furthermore LNAME was capable of attenuating the quinolinic acid-induced reductions in viability in human astrocytes (Braidy et al., 2009a).

Pierozan et al. (2015) have reported that quinolinic acid at concentrations between 10 and $100 \mu \mathrm{M}$ with exposure times ranging from 24 to $72 \mathrm{~h}$ failed to affect the viability of primary rat astrocytes or neurons. These are consistent with results from our own laboratory where quinolinic acid in either the presence of absence of glycine at doses ranging from 0.1 to $100 \mu \mathrm{M}$ over $24 \mathrm{~h}$ failed to affect the viability of rat primary cortical neurons. Some metabolites are likely to contribute to neuronal cell death to a greater degree than others and this may have implications with regard to underlying mechanisms associated with the influence of the KP in neurodegenerative disorders.

\subsection{The effects of KP metabolites on neurite outgrowth and complexity}

KP metabolites have the potential to influence neuronal transmission by affecting neuronal integrity via reducing viability, neuritic complexity or plasticity. The number, length and complexity of dendrites is widely believed to underlie neuronal interaction and network integration corresponding to key aspects of brain function such as cognitive function (Ferrante et al., 2013). As such, in vitro experiments which assess the impact of KP metabolites on neurite outgrowth and neuronal complexity are of interest. Exposure of rat primary striatal neurons to quinolinic acid at 10,25 and $100 \mu \mathrm{M}$ has been reported to result in hyperphosphorylation of neurofilament subunits which is believed to be mediated by NMDA receptor-driven increases in intracellular calcium. In addition, this group demonstrated that these same concentrations of quinolinic acid reduced the neurite length of rat primary striatal neuronal cells in culture. It was proposed that quinolinic acid-induced neuronal injury interferes with the physiological roles of the axonal cytoskeleton in mediating neuronal outgrowth (Pierozan et al., 2015). This same group had previously reported similar results in striatal cortical slices from young rats following an infusion of quinolinic acid into the brain where the hyperphosphorylation of neurofilaments was prevented by the NMDA receptor antagonist, MK-801, or the iNOS inhibitor, L-NAME (Pierozan et al., 2010). Moreover, we have also found that 3hydroxykynurenine $(0.1 \mu \mathrm{M})$ and to a lesser extent 3hydroxyanthranilic acid $(0.1 \mu \mathrm{M})$ reduce measures of neuronal outgrowth and complexity of rat primary cortical neurons including the number of primary neurites, neurite length and the number of branching intersections at various distances from the cell soma. In addition, we have also found KYNA to reduce measures of neuronal outgrowth and complexity at concentrations ranging from 0.1 to $10 \mu \mathrm{M}$ (unpublished data). This is in accordance with the literature in that KYNA is believed to play a dual role with opposing effects depending on whether it is present in nanomolar or micromolar concentrations (Rozsa et al., 2008).

Variations in the literature regarding the effects that KP metabolites have on neuronal complexity may be as a consequence of variations between experimental parameters. These may include differences in the treatment duration and concentration, variations in protocols for the preparation of primary cultures and also the specific type of neuron utilised. In particular the age at which the neuronal cultures are treated may greatly influence the outcomes and cellular resilience or lack thereof to exposure to the various metabolites. Verstraelen et al. (2014) have recently described the maturation of primary murine hippocampal neurons and illustrate the changes in morphological and electrophysiological properties that occur with respect to the age of the cells in culture. In addition they showed that the balance between KYNA and quinolinic acid was critical for the maintenance of the activity of hippocampal neuronal networks, with either quinolinic acid or KYNA alone disturbing the synchronous burst firing of these networks, while consecutive addition of the NMDA receptor modulators allowed for normal network activity.

Macrophages of the murine cell line, BAC1.2F5 stimulated with IFN- $\gamma$ release nitric oxide alongside 3-hydroxykynurenine and quinolinic acid into the culture media. These effects may be blocked by treatment with the IDO inhibitor, norharmane (Chiarugi et al., 2000). Circulating or infiltrating macrophages act as a peripheral source of KP metabolites. Circulating metabolites may in turn gain access to the brain which provides a putative link between systemic immune activation and inflammation influencing neuronal transmission. It is now well recognised that bidirectional communication occurs between the CNS and the immune system. This is achieved through cytokines accessing the brain directly via circumventricular organs, or being transported across the BBB by their respective active transporters or infiltrating immune cells. In addition cytokines can act via the vagus nerve to alter neurotransmission in the brain. A role for the peripheral immune system in neuroinflammatory processes underlying the pathophysiology of neurological disorders is well accepted and reviewed extensively elsewhere [see, Najjar et al. (2013), Haroon et al. (2012), Glass et al. (2010) for review]. As such, direct activation of the KP centrally, through locally produced or infiltrating cytokines may therefore impact upon neuronal transmission through the aforementioned actions of KP metabolites. Additionally, activation of the KP peripherally may influence neuronal transmission as some KP metabolites gain access to the brain (Fig. 1). Thus, KP-mediated alterations in neuronal transmission and integrity may be one such mechanism by which stress could contribute to the development of symptoms of neuropsychiatric and degenerative disorders.

\section{A role for the KP in the pathophysiology of neurodegenerative disorders}

The KP is appreciated as having an important part to play in the development of multiple neurodegenerative disorders including 
Parkinson's disease, Alzheimer's disease, Huntington's disease, amylotrophic lateral sclerosis (ALS), multiple sclerosis (MS), epilepsy and stroke (Lim et al., 2010; Anderson and Rodriguez, 2011; Maddison and Giorgini, 2015; Bohar et al., 2015; Cuartero et al., 2014; Tan et al., 2012).

A role for IDO-related activation of the KP in the pathophysiology of neurological disease and psychiatric disorders has been examined extensively over the past decade and is reviewed elsewhere (Myint and Kim, 2014; 2003; Schwarcz et al., 2012b; Maes et al., 2011). The extent to which TDO-related activation of the KP is involved is yet to be fully investigated and understood. Apart from early work carried out by Curzon and Green (1969), demonstrating that psychophysiological stress in rats induces hepatic TDO, little has been done to assess in greater detail the degree to which TDO-related activation of the KP may be implicated in the cause or progression of stress-associated disorders.

\subsection{A role for the KP in Parkinson's disease}

Endogenous excitotoxic mediators have been implicated in the degeneration of nigral dopaminergic neurons in Parkinson's disease for some time now. Parkinson's disease patients show increases in 3-hydroxykynurenine in the frontal cortex, putamen, and the substantia nigra pars compacta, while KYNA concentrations were found to be decreased (Ogawa et al., 1992). A widely used mouse model of Parkinson's disease which involves the administration of 1-methyl-4-phenyl-1,2,3,6-tetrahydropyridine (MPTP) demonstrated a reduction in KAT I expression in these mice (Knyihar-Csillik et al., 2004). Similarly KAT II activity was reported to be reduced in cortical slices of rats treated with $\mathrm{MPP}^{+}$, the toxic metabolite of MPTP (Luchowski et al., 2002). Modulation of the KP, in terms of shifting its activation towards KYNA production, has been suggested as a putative novel approach for the development of neuroprotective treatments for use in Parkinson's disease (Zadori et al., 2011, 2013).

The development of a BBB permeable KMO inhibitor provides a novel strategy that may help to reduce the toxic effects associated with activation of the KP in neurodegenerative processes. Several studies have shown that NMDA receptor antagonists can exert neuroprotection in both in vitro and in vivo models of Parkinson's disease [see, Zinger et al. (2011) for review]. Furthermore, intracerbroventricular administration of nicotinylalanine, a KMO and KYNU inhibitor, alongside systemic administration of kynurenine and probenecid, an inhibitor of KYNA removal from the cerebrospinal fluid, leading to increased levels of KYNA in the brain, has been reported to block quinolinic acid-mediated toxicity within the substantia nigra in rats (Miranda et al., 1997). In addition, nicotinylalanine and the KMO inhibitor, Ro-618048 were capable of reducing dyskinesia associated with long term L-Dopa usage. This was achieved without altering its beneficial effects in monkeys who had previously received MPTP and developed moderate to severe Parkinsonian syndrome (Gregoire et al., 2008). Furthermore, systemic administration of kynurenine and probenecid prevented reductions in striatal dopamine levels, striatal neurodegeneration and increased rotation behaviour, a marker of dopaminergic system alterations in the 6-hydroxydopamine model of Parkinson's disease (Silva-Adaya et al., 2011). Thus far it has been proposed that KYNA, or KYNA derivatives that have a higher propensity to cross the BBB would be useful in aiding neuroprotective processes in Parkinson's disease patients. Similarly, it is believed that kynurenine derivatives and various KP inhibitors such as, the KMO inhibitor, Ro618048 , would be a strategic way of ameliorating KYNA deficits observed in Parkinson's disease patients by way of increasing the levels of available kynurenine for KYNA synthesis (Nemeth et al., 2006). In particular, melatonin production which provides protection against various Parkinsonian toxins including, MPTP and 6-hydroxydopamine (Sharma et al., 2006; Patki and Lau, 2011), is reduced by the presence of increased glial TDO or IDO activation. Administration of melatonin may thus act as a complementary adjunctive therapeutic strategy in Parkinson's disease (Anderson and Maes, 2014).

\subsection{A role for the KP in Alzheimer's disease}

Alterations in the KP have also been observed in Alzheimer's disease including a reduction in the serum concentrations of tryptophan and KYNA and increases in kynurenine and 3hydroxykynurenine, with these concentrations correlating with the level of cognitive impairment [see, Ting et al. (2007) for review]. In contrast, an increase in the concentrations of KYNA has been reported in the striatum of post mortem brains of Alzheimer's disease patients which is proposed to be a compensatory mechanism in response to a hyperactive striato-frontal loop due to neuronal loss in cortical areas (Baran et al., 1999). In conjunction with the increase in KYNA, $\beta$-amyloid, specifically A $\beta 1-42$ has been reported to increase quinolinic acid production to neurotoxic concentrations in both human macrophages and microglia which may contribute to oxidative processes in Alzheimer's disease (Guillemin et al., 2003b). Similarly A $\beta 1-42$-induced increases in IDO mRNA expression, allows for 3-hydroxykynurenine- and 3-hydroxyanthranilic acid-induced oxidative stress which could contribute to neurodegeneration (Guillemin et al., 2003b). A role for the KP has been proposed as a consequence of both TDO and IDO induction ( $\mathrm{Wu}$ et al., 2013). A recent study has indicated that TDO-mediated KP activation may be important in the formation of neurofibrillary tangles and may be associated with senile plaque development. This study demonstrated that TDO is highly expressed in the brains of both Alzheimer's disease patients and the 3xTg mouse model of Alzheimer's disease. Moreover TDO co-localises with quinolinic acid, neurofibrillary tangles and amyloid deposits in the hippocampus of post mortem brains of Alzheimer's disease patients. It is proposed that activation of TDO in this instance is driven by prolactin and cortisol (Wu et al., 2013). With regards to potential therapeutic targets, a small molecule KMO inhibitor, JM6, was shown to be capable of reducing extracellular glutamate centrally and increasing KYNA concentrations in the brain and serum of rats following chronic oral administration. In addition, it was also capable of preventing spatial memory deficits, anxiety-like behaviour and synaptic loss, while having no effect on amyloid $\beta$ accumulation in a mouse model of Alzheimer's disease associated with over-expression of the human amyloid precursor protein (Zwilling et al., 2011).

\subsection{A role for the KP in Huntington's disease}

Metabolites of the KP have been implicated in the pathophysiology of Huntington's disease. Both Huntington's disease patients and transgenic mice expressing the mutant huntingtin gene are reported to have increased concentrations of quinolinic acid and 3hydroxykynurenine in the neostriatum and cortex (Guidetti et al., $2004,2000)$. In the fruit fly model of this disorder, genetic inhibition of either TDO or KMO resulted in a decrease in the levels of 3hydroxykynurenine and a shift towards KYNA synthesis and neuroprotection. Further effects of KP metabolites in Huntington's disease are reviewed elsewhere (Tan et al., 2012; Bohar et al., 2015). Various KMO inhibitors including JM6 and Ro61-8048 were reported to attenuate neurodegeneration, with JM6 preventing synaptic loss, stabilising synaptic activity and reducing neuroinflammation in a mouse model of Huntington's disease (Tan et al., 2012; Amaral et al., 2013). 


\subsection{A role for the KP in ALS}

The KP has also been directly linked with ALS as patients were reported to have increased cerebrospinal fluid and serum concentrations of kynurenine and quinolinic acid. KP activation could not be specifically attributable to either IDO or TDO induction alone, however the authors propose that considering the higher expression levels of IDO in the brain relative to TDO, in this instance, IDO was driving the changes in metabolite concentrations (Chen et al., 2011). In vitro studies however, in the mouse motor neuron cell line NSC-34, have found that both control and IFN- $\gamma$-stimulated cells stain positively for TDO, while IDO was only found in the IFN$\gamma$-treated group (Chen et al., 2011). This is a conflicting finding, in that IFN- $\gamma$ was shown to induce TDO and furthermore that the reciprocal relationship that was previously reported (Guillemin et al., 2007) to exist between TDO and IDO in both primary human neurons and the human neuronal cell line, SK-N-SH, is absent in these cells, thus highlighting differential enzyme expression patterns between mice and humans (Guillemin et al., 2007). Other indirect ways by which the KP could be associated with the pathology of ALS include the excitotoxic action of quinolinic acid at the NMDA receptor and also by raised synaptic glutamate concentrations which has been implicated in the pathophysiology of ALS. In fact, interference in glutamate regulation, through the use of riluzole which inhibits hippocampal glutamate release (Martin et al., 1993), is the only current treatment available for ALS. Moreover various KP metabolites are capable of producing free radicals and driving oxidative stress which is an additional putative contributing factor to ALS (Chen and Guillemin, 2012).

\subsection{A role for the KP in $M S$}

Considering MS is a neuroinflammatory disorder all of the work done to date has primarily focused on IDO-driven KP activation, with little to no data available on a companion role for TDO (Rajda et al., 2015; Lim et al., 2010; Chen and Guillemin, 2009; Anderson and Rodriguez, 2011). Irrespective of this, there are both preclinical and clinical studies, recently reviewed by Rajda et al. (2015), illustrating how activation of the KP is associated with neuronal degeneration in both MS patients and animal models of MS. Both 3hydroxykynurenine and quinolinic acid concentrations have been reported to be increased in the spinal cord of rodents subjected to experimental autoimmune encephalitis (EAE), with increases being attenuated by systemic treatment with the KMO inhibitor, Ro618048 (Chiarugi et al., 2001). Moreover, quinolinic acid has been shown to be capable of inducing neuronal, astroglial and oligodendroglial cell death (Braidy et al., 2009b; Sundaram et al., 2014). Furthermore, circulating KYNA concentrations in patients varies depending on the phase of the disease. Low levels have been detected in the cerebrospinal fluid of patients in remission (Rejdak et al., 2002), while high levels were reported in the cerebrospinal fluid of patients experiencing acute relapse (Rejdak et al., 2007). Therapeutic strategies targeting the KP in MS primarily aim to increase the concentrations of KYNA in the brain by treating with KMO inhibitors in order to shift metabolism on the KP towards conversion of kynurenine to KYNA [see, Rajda et al. (2015) for review].

\subsection{A role for the KP in seizure control}

In relation to epilepsy and seizures, evidence to date only goes so far as to implicate IDO-mediated KP activation with suggestions of a potential role for TDO being overlooked (Xie et al., 2014; Anderson and Rodriguez, 2011). Recently a genetic link between the KP gene, aminocarboxymuconate semialdehyde decarboxylase
( $A C M S D)$, which encodes the enzyme that drives the metabolism of 2-amino 3-carboxymuconate 6-semialdehyde to 2-aminomuconic acid 6-semialdehyde rather than quinolinic acid, and familial myoclonic epilepsy has been identified. This stop codon mutation is implicated in ACMSD deficiency, resulting in quinolinic acid accumulation in the brain and in the onset of epilepsy (Marti-Masso et al., 2013). In addition, it was found that administration of kynurenine in combination with probenecid which prevents it from being removed from the CNS, protected against pentylenetetrazol-induced seizures in rats (Nemeth et al., 2004).

\subsection{A role for KP activation in ischaemic stroke}

The KP has been implicated in cardiovascular disease and stroke (Nikkheslat et al., 2015; Cuartero et al., 2014; Darlington et al., 2007). A recent study by Cuartero et al. (2014) was aimed at defining a role for the aryl hydrocarbon receptor (AhR) in stroke. Kynurenine, a known agonist at the AhR (Opitz et al., 2011) mediates AhR activation in times of ischemia. In this investigation the authors reported that ischemic insult led to increases in the expression of the AhR in the brain following middle cerebral artery occlusion in mice. The effects were abolished in $A h R$ knockout (-/-) mice or following systemic administration of the AhR antagonist, 6,2' $4^{\prime}$-trimethoxyflavone (TMF) indicating that the AhR mediates acute ischemic damage. AhR activation in neurons inhibits cAMP response element binding (CREB) protein, it is proposed that via this inhibition, ischemia and AhR activation inhibit pro-survival pathways. With regards to a role for kynurenine in this instance, Cuartero et al. (2014) reported that the concentrations of kynurenine increase in the brain as early as $3 \mathrm{~h}$ following middle cerebral artery occlusion and remain elevated at $24 \mathrm{~h}$ post occlusion in mice. Interestingly, this increase occurred only in the brain and was as a consequence of ischemia increasing the expression of TDO rather than IDO or IDO-2 in both the core and peri-infarct regions. Furthermore this group demonstrated that inhibition of TDO but not IDO reduced the infarct volume relative to control. Similarly they reported that TDO inhibition prevented middle cerebral artery occlusion-induced increases in concentrations of kynurenine in the brain resulting in an accumulation of central tryptophan and reduced activation of the AhR. Overall this study suggests that TDO-mediated kynurenine agonism at the AhR and concomitant inhibition of pro-survival pathways in neurons is a potential mechanism by which tryptophan metabolism may mediate ischemic damage. This highlights TDO as the primary enzyme responsible for kynurenine production following stroke, and identifies TDO as an important therapeutic target with regards to preventing ischemic damage. In contrast other studies have found that administration of kynurenine prior to ischemic damage has a protective effect due to the action of the downstream metabolite, KYNA (Gigler et al., 2007). Gigler et al. (2007) used the middle cerebral artery occlusion in mice as a model for focal cerebral ischemia, while bilateral carotid artery occlusion in gerbils was used to examine global cerebral ischemia. Intraperitoneal administration of L-kynurenine sulphate 15 min prior to middle cerebral artery occlusion significantly reduced the cortical infarct surface area at all doses tested. This reduction in cortical infarct surface area was also achieved by pretreatment with lower doses of the anti-ischemic agent and AMPA receptor antagonist, GYKI53655. In addition, administration of L-kynurenine sulphate or GYKI53655 $2 \mathrm{~h}$ prior to a transient bilateral carotid artery occlusion prevented pyramidal neuronal loss in the CA1 region of the hippocampus of these gerbils. Furthermore, both L-kynurenine sulphate or GYKI53655 attneuated ischemia-induced deterioration of spatial memory and hypermotility as assessed by spontaneous alternation and locomotor activity in the Y-maze respectively. 


\section{A role for the KP in the pathophysiology of neuropsychiatric disorders}

In relation to psychiatric disorders, it has long been acknowledged that the KP plays a role in the pathophysiology of mood disorders, anxiety and schizophrenia (Oxenkrug, 2010; Schwarcz et al., 2012a). It is well established that genetic pre-disposition and brain development in early life are factors which can increase a person's vulnerability to the emergence of psychiatric symptoms in later life. The KP may play a role in mediating such effects.

Single nucleotide polymorphisms (SNPs) in tdo2, the gene which encodes TDO such as, rs3775085, A/C; rs3755907, A/G; rs3755908, $\mathrm{C} / \mathrm{T}$, have been associated with autism, attention deficit hyperactive disorder and Tourette syndrome (Comings, 2001; Nabi et al., 2004). Similarly preliminary data from a genome wide association study identified two SNPs, rs2929115 and rs2929116, in the IDO gene with this genetic variation in ido and ido-2 proposed to contribute to variations in outcome following antidepressant treatment (Cutler et al., 2012). Moreover, in the mouse nervous system Kanai et al. (2009b), identified the expression of TDO in the brain, with high levels of its expression in the hippocampus and cerebellum and lower levels in the cerebral cortex, striatum and midbrain. In addition, this group identified variants of the tdo gene, namely tdo variant 1 and tdo variant 2 and they showed that the TDO protein encoded by the $t$ do gene shares similar specific enzymatic activity to the proteins encoded by the novel variants. In addition, the group demonstrated that these variants exhibit altered expression patterns which fluctuate from postnatal day $1-49$. As a consequence of this expression pattern, the authors suggest that $t d o$ full-form is an important modulator of hippocampal development, while both it and tdo variant 2 are important in early cerebellar development (Kanai et al., 2010). In adult mice TDO was demonstrated as having an important role in the maintenance of brain morphology as $t d o-/$ - mice were shown to have increased neurogenesis and these mice exhibited a reduced anxiety-related phenotype when subjected to the elevated plus maze and open field test. These knockout mice exhibited increased plasma levels of tryptophan, kynurenine and the serotonin metabolite, 5-hydroxyindoleacetic acid, as well as increased brain levels of tryptophan, serotonin and 5hydroxyindoleacetic acid (Funakoshi et al., 2011b; Kanai et al., 2009a). In contrast, ido - /- mice have only recently come into use to facilitate an assessment of KP activation in animal models of psychiatric disorders (Heisler and O'Connor, 2015). A recent study demonstrated that exposure to lipopolysaccharide (LPS) increased plasma and brain kynurenine levels and led to reduced time exploring a novel object in wild type mice. However, the same LPS treatment in either ido $-/$ - or kmo $-/-$ mice does not have the same physiological or behavioural effects. This study implicates KP activation in driving these deficits in object recognition memory which the authors propose may contribute to cognitive dysfunction in inflammation-induced depression (Heisler and O'Connor, 2015).

Development of the brain can be influenced by early life adversity including exposure to infectious agents, activation of the immune system and exposure to stress. A number of studies have been carried out to date which assess a role for the KP in driving developmental processes (Khalil et al., 2014; Pisar et al., 2014; Forrest et al., 2013b). These studies suggest that activation of the KP as a consequence of prenatal stress and inflammation may have the potential to contribute to alterations in the development of the nervous system. Preclinical models, such as poor maternal care, demonstrate that early life adversity has the potential to alter brain development [see, Lucassen et al. (2013), McEwen (2008) for review]. In early life, tryptophan is an important factor involved in the development of regions of the brain associated with psychiatric disorders. This may be due to the fact that it is the precursor for serotonin which has been shown to exert effects in the development of target tissues [see, Lauder (1990) for review] alongside altering the expression of brain derived neurotrophic factor (BDNF) (Vaidya et al., 1997). The identification of fluctuations in the expression of TDO mRNA and its variants in both the developing and adult brain suggests that TDO is important in the regulation of the development of specific brain regions such as the hippocampus and cerebellum (Kanai et al., 2010). Thus, disruptions in TDO expression may be responsible for subsequent behavioural changes, such as the emergence of anxietyrelated behaviour in adult life (Funakoshi et al., 2011a). Tryptophan restriction in rats during an early postnatal stage was shown to result in reduced levels of serotonin in the brain alongside reduced dendritic arborisation, spine density and cell proliferation in the dentate gyrus of the hippocampus. These rats also displayed anxiety-like and depressive-like behaviour, however this study failed to examine a role for KP activation in mediating these morphological and behavioural changes (Zhang et al., 2006).

$\mathrm{KP}$ activity has been implicated in normal brain development (Forrest et al., 2013a, 2013b; Pisar et al., 2014). Prenatal inhibition of the KP downstream of TDO/IDO, using the KMO inhibitor, Ro618048 resulted in rapid changes in protein expression. For instance, the levels of NMDA receptor subunits GluN2A and GluN2B were decreased and increased respectively in the brains of embryos $5 \mathrm{~h}$ post Ro61-8048 administration. In 21 day old offspring, there was increased neurogenesis, neuronal excitability and paired-pulse firing, alongside increased long term potentiation and protein expression of GluN2A, GluN2B and postsynaptic density 95. These changes highlight that inhibition of activation of the neurotoxic arm of the KP prenatally affects neuronal development of offspring assessed at weaning [postnatal day 21] (Forrest et al., 2013a, 2013b). In addition, prenatal administration of Ro61-8048, which increases the levels of kynurenine and KYNA in the brain of embryos (Forrest et al., 2013a), altered dendritic parameters in the hippocampus such as increasing the radial intersections at various distances from the cell body (Pisar et al., 2014). It is proposed that such changes may be as a consequence of altered NMDA receptor expression at the various stages of development (Forrest et al., 2013b). Another study which involved the administration of kynurenine to cultured murine astrocytes has been shown to induce the expression of nerve growth factor, which the authors propose may be a mechanism by which circulating tryptophan and kynurenine may exert trophic support (Dong-Ryul et al., 1997).

Furthermore, clinical studies suggest that early life adversity and subsequent neurodevelopmental abnormalities and alterations in the stress response, such as HPA axis hyperactivity can persist during adulthood [see, Faravelli et al. (2012) for review]. Reductions in the levels of GR expression were found in the post mortem brains of patients with major depression, bipolar disorder and schizophrenia, indicating altered GR functioning (Webster et al., 2002). Similarly, findings reviewed by Holtzman et al. (2013) show that schizophrenic patients exhibit a hyperactive HPA axis as well as dexamethasone non suppression in the dexamethasone suppression test. This re-programming of the HPA axis and associated blunted GR signalling in immune cells, is believed to be an important link between prenatal stress and risk of psychosis in later life (Koenig et al., 2002). How these early life stress-related physiological changes affect KP activation both peripherally and centrally in the long term is yet to be examined, with interesting findings having emerged over the past decade on the role for KP activating in psychiatric disorders which will be discussed hereafter.

\subsection{A role for KP activation in major depression}

Several studies have identified increases in circulating kynurenine and decreases in circulating tryptophan concentrations 
culminating in alterations to the kynurenine over tryptophan ratio, which is commonly used as an indicator of KP activation. Increases in the concentrations of kynurenine, quinolinic acid and KYNA have been identified in the cerebrospinal fluid (Raison et al., 2010), while decreases in plasma KYNA concentrations have also been reported in depressed patients (Myint et al., 2007). These changes indicate that the KP may be influenced in specific subtypes of depression [see, Reus et al. (2015); Lopresti et al. (2014) for review]. A role for inflammation in the pathogenesis of depression was originally put forward by Smith (1991) who developed the macrophage theory of depression in which he emphasised that it was the excessive secretion of cytokines from macrophages which bring about depression. Support for this came from the positive correlation between increased inflammatory cytokines in the plasma of chronic depressives and the finding that depression developed in hepatitis $C$ patients receiving interferon (IFN) $\alpha$ treatment (Raison et al., 2005). As such, secondary depression which occurs as a consequence of infection, inflammation or oxidative stress may be more so associated with IDO-driven KP activation (Fukuda, 2014). Reactive depression is associated with exposure to major life stressors, HPA axis activation and dysregulation (Fukuda, 2014) and likely induction of TDO and KP activation. While these links between stress and KP activation have not much been explored in major depression to date, studies in animal models of stress lend support to the likely role of these interactions in major depressive illness. Early investigations by Green and Curzon (1968), reported that administration of the stress hormone, hydrocortisone to rats resulted in increased TDO activity and reductions in brain serotonin concentrations which were preventable by prior treatment with the TDO inhibitor, allopurinol. Furthermore, periods of immobilisation stress increased hepatic TDO activity which was prevented by adrenalectomy, indicating that stress-related TDO induction is mediated by HPA axis activation (Curzon and Green, 1969).

More recently Gibney and co-workers demonstrated a role for TDO in driving immobilisation stress-induced depressive-like behaviour in rats as indicated by an increase in immobility time in the forced swimming test (FST). Rats exposed to immobilisation stress showed elevated levels of hepatic TDO expression and activity, an increase in the kynurenine over tryptophan ratio in tandem with depression-related immobility in the FST. Prior treatment with the TDO inhibitor allopurinol attenuated the stress-related increase in immobility in tandem with a reduction in the KP activation ratio (Gibney et al., 2014). Similarly, IDO activation has been shown to mediate LPS-driven depressive-like behaviours in rodents, with these behaviours being abolished by use of the IDO inhibitor, 1-methyl tryptophan (L) (O'Connor et al., 2009; Lawson et al., 2013). O'Connor et al. (2009), demonstrated that peripheral administration of LPS to mice increased IDO mRNA expression in the brain alongside an increase in the kynurenine over tryptophan ratio in both the brain and plasma. In addition, these mice displayed increased immobility time in both the FST and tail suspension test, which is indicative of depressive-like behaviour. These LPS-induced changes in measures of KP activation and behaviour were abolished by pre-treatment with 1-methyl tryptophan (L) or minocycline, an inhibitor of microglial activation. Furthermore Lawson et al. (2013) reported that intracerbroventricular administration of LPS to mice resulted in increased kynurenine over tryptophan ratio in the brain, alongside anhedonic and depressive-like behaviour as measured by the sucrose preference test and tail suspension test respectively. This anhedonic phenotype was absent in ido - / - mice treated with LPS, and similarly both behavioural changes were prevent by administration of a racemic mixture of 1-methyl tryptophan in conjunction with LPS. Despite the fact that tryptophan metabolism associated with KP activation may conceivably lower tryptophan availability for serotonin synthesis, there is inconsistent evidence for this occurring following administration of either LPS, polyinositolphosphatidic acid (poly I:C) or inflammatory cytokines to rodents. As such it is believed that it is the production of KP metabolites which drive the depressive-like behaviour in animal models (Gibney et al., 2013; Myint et al., 2007; Dunn et al., 2005).

There is a large body of literature demonstrating that exercise is effective in alleviating suffering from mood disorders [see, Powers et al. (2015) for review]. A recent study by Agudelo et al. (2014) in mice suggests that exercise-driven kynurenine metabolism in skeletal muscle mediates resilience to stress-induced behaviour associated with depression. Brain penetrant kynurenine may be converted to various neuroactive metabolites which have been implicated in the pathophysiology of a number of brain disorders including depression. Conversion of kynurenine to kynurenic acid by KATs in the periphery however limits its delivery to the brain as kynurenic acid is incapable of crossing the BBB. Agudelo and colleagues have identified a molecular axis in mice whereby increasing the activity of the transcriptional co-activator PGC-1 11 in skeletal muscle mediates resilience against stress-induced depression-related behaviours and stress-related deficits in the expression of a range of molecular markers of neurotrophy. In conjunction with PGC- $1 \alpha 1$ the transcription factors PPAR $\alpha / \delta$ induce the expression of KATs to reduce levels of kynurenine which reach the brain, consequently impeding the conversion of kynurenine in the brain to active metabolites associated with altered neurotransmission and behaviour. Linking peripheral kynurenine metabolism to stress resilience is further supported by the induction of PGC-1 11 and up-regulation of KAT in skeletal muscle and increase in circulating kynurenic acid concentrations in response to free wheel running. Moreover aerobic exercise was also reported to increase PGC-1 $\alpha 1$ and KAT enzyme expression in human biopsied muscle tissue. Exercise may thus act as a key adjunct in treating stress-related disorders like depression through limiting kynurenine's access to the brain. The clinical significance of the effect of exercise on PGC-1 $\alpha 1$ is likely to be of greatest significance where expression of the transcriptional co-activator is reduced. Skeletal muscle PGC- $1 \alpha 1$ expression is known to decline with age and in individuals with type 2 diabetes, and increased kynurenine accumulation in the brain may be a potential contributor to co-morbid depression. This proposed mechanism also provides a platform from which new treatments can be investigated and developed where mediators of the skeletal muscle pathway are harnessed to provoke exercise-like responses with regard to stress resiliency. Further evaluation of serum biomarkers in human studies would be favourable to demonstrate reductions in circulating kynurenine and concomitant increases in KYNA following exercise training.

There is evidence emerging to support a contributing role of $\mathrm{KP}$ activation, which may be driven by the induction of TDO and IDO in tandem or isolation, with the relative contribution of either being determined by the presence or absence of inflammatory factors, the nature and duration of the stressor exposure, and whether these two elements are present alone or in combination (Gibney et al., 2014).

\subsection{A role for KP activation in anxiety disorders}

Information regarding the HPA axis in all anxiety diagnostic subgroups is scarce and inconsistent, with neither early stressful life events nor HPA axis dysfunction being unique to any diagnostic group [see, Furtado and Katzman (2015), Faravelli et al. (2012) for review]. Furthermore, alterations in circulating inflammatory cytokines in these patients is equivocal. A meta-analysis examining inflammatory markers in obsessive compulsive disorder (OCD) patients reported reduced levels of circulating IL- $1 \beta$ and IL-6 and suggests that inconsistencies within the literature are as a 
consequence of confounding factors such as varied medication and co-morbidity with depressive disorders (Gray and Bloch, 2012). Men suffering from generalised anxiety disorder and panic disorder have been reported to exhibit raised circulating concentrations of C-reactive protein (Vogelzangs et al., 2013). Similarly, patients suffering from post-traumatic stress disorder (PTSD) showed increased inflammatory markers (Spitzer et al., 2010). Consequently, a lack of clarity regarding changes in HPA axis and immune function parameters in these patients precludes indicating a role for the kynurenine pathway which may be affected in these patients, whether it be over-activated and whether it is TDO or IDO driving this activation. Thus far, little has been reported with supporting evidence for KP activation in any specific diagnostic subgroup. Maes et al. (2002) demonstrated that women in the early postpartum period have increased plasma kynurenine concentrations and kynurenine over tryptophan ratio associated with increased serum levels of IL-6 and IL-8. This group also found significant positive associations between changes in kynurenine pathway metabolite concentrations and depressive and anxiety symptoms in these women during the post-partum period.

Although various animal models exist for OCD, PTSD and panic disorders, assessment of KP activation has not been carried out in these models to date [for review of these models, see Goswami et al. (2013), Johnson and Shekhar (2012), Albelda and Joel (2012)]. Limitations arise with the tests used for assessing anxiety-like behaviour in rodents, as they are not specific to any one anxiety-related disorder (Deacon, 2013). Preclinical studies have found associations of anxiety-related behaviours with altered KP activation in both mice and rats (Gibney et al., 2013; Laugeray et al., 2011). Laugeray et al. (2011) demonstrated in mice exposed to the unpredictable chronic mild stress paradigm, the elevated plus maze and FST, an elevated lung kynurenine over tryptophan ratio and reduced levels of KYNA in the amygdala were associated with anxiety- and depressive-like behaviours. Moreover, Gibney et al. (2013) administered Poly I:C to rats systemically and showed increased IDO mRNA expression and kynurenine to tryptophan ratio in the brain was associated with anxiety-related behaviour as assessed in the open field test. In addition, postnatal administration of LPS to male rats, stimulates KP activation with increased concentrations of tryptophan, kynurenine, 3hydroxykynurenine and quinolinic acid, but not KYNA, in different brain regions as well as in plasma (Kubesova et al., 2015). Sominsky et al. (2013) showed using a lower dose of LPS and a similar experimental design that female rat pups exposed to LPS exhibit anxiety-like behaviour in adult life. It was also reported that systemic administration of L-kynurenine provokes anxiety-like behaviour and impaired object recognition memory in mice (Varga et al., 2015). This was proposed to be as a consequence of dose-dependent increases in KYNA concentrations in the striatum following systemic administration of L-kynurenine sulphate (Swartz et al., 1990). In such investigations KP activation would mostly likely be mediated by inflammatory cytokines and the induction of IDO rather than changes to TDO activity. In early life, stress associated induction of TDO and KP activation may be a mechanism which links HPA axis dysregulation to changes in CNS circuitry within mood and anxiety associated brain regions. A study involving $t d o-/-$ mice reported reduced anxiety-related behaviour in two anxiety behavioural tests, the elevated plus maze and open field test when compared to the wild type control strain (Kanai et al., 2009a). This was proposed to be as a consequence of increased concentrations of serotonin in the hippocampus and midbrain implicated in neural development through proneurogenic and trophic actions [see, Gaspar et al. (2003) for review]. Furthermore, maternal separation, a widely used model of early life stress, in mice was shown to result in an increase in the ratio of 3-hydroxykynurenine to KYNA in the prefrontal cortex. These changes were accompanied by altered emotional responses such as, reductions in emotional memory, depressive-like behaviour and anxiety-like behaviour as measured by the passive avoidance, tail suspension and open field tests respectively. These studies involved the use of both male and female mice, and demonstrated that behavioural abnormalities were more severe in females than males (Gracia-Rubio et al., 2015).

\subsection{A role for KP activation in schizophrenia}

In contrast to depressed patients, schizophrenic patients show a shift in the KP towards increases in serum and cerebrospinal fluid concentrations of KYNA [see, Muller and Schwarz (2010) for review]. A study done by Chiappelli et al. (2014) investigating schizophrenic patients' response to stress, showed that these patients have a higher rate of distress intolerance. Furthermore it showed that stress increased salivary KYNA in both patients and controls, the levels of which were associated with the severity of clinical symptoms in patients. Reduced KMO activity has also been reported in the post mortem brains of schizophrenic patients (Wonodi et al., 2011).

Alterations in the regulation of the KP in schizophrenia may be as a consequence of an alteration in immune activation. This change appears to involve a shift from a type 1 immune response, which involves the production of IL- 2 and IFN $\gamma$ by $\mathrm{Th}_{1}$ cells, M1 macrophages and monocytes which mediates a cellular immune response, towards a type 2 immune response, which involves the production of IL- 4 and IL- 10 by $\mathrm{Th}_{2}$ cells and M2 macrophages or monocytes which drives the humoral immune response [see, Muller and Schwarz (2010) for review]. A type 1/type 2 immune imbalance may result in a reduction in central and peripheral IDO activation, however tryptophan-induced TDO activation remains intact. In fact, TDO has been found to be up-regulated in the post mortem brains of schizophrenic patients and this appears to be astrocyte specific (Miller et al., 2004). This type 1/type 2 shift hypothesis as a pathophysiological mechanism for the development schizophrenia was contradicted by Kim et al. (2009). This group demonstrated that drug-naïve schizophrenic patients exhibited increased levels of IFN $\gamma$, a $\mathrm{Th}_{1}$-specific cytokine and reduced levels of IL-4, a $\mathrm{Th}_{2}$-specific cytokine in the plasma. It was proposed that treatment with certain antipsychotics may contribute to this shift in the immune response as seen with risperidone (Cazzullo et al., 2002). Furthermore, plasma tryptophan concentration in drugnaive schizophrenic patients were negatively associated with the total positive symptoms score. This was proposed to be as a consequence of either tryptophan availability for serotonin metabolism, or tryptophan metabolism to neuroactive KP metabolites (Kim et al., 2009). A recent study by Schwieler et al. (2015), demonstrated that olanzapine-treated patients suffering from chronic schizophrenia or schizoaffective disorder, have increased levels of IL-6 in the cerebrospinal fluid. This increase in IL-6 was positively correlated with an increase in the KYNA levels in the cerebrospinal fluid. Moreover, increased levels of KYNA have been found in astrocyte-dense regions of the brain associated with the pathology of schizophrenia, including Brodmann area 9 and the anterior cingulate cortex (Schwarcz et al., 2001). In contrast Myint et al. (2011) demonstrated a decrease in the levels of KYNA and an increase in the levels of 3-hydroxykynurenine in the plasma of medication-naïve/free schizophrenic patients. This KP imbalance was partially reversed following 6 week treatment with either risperidone, amisulpride, olanzapine or aripiprazole. In this instance, activation of the neurotoxic arm of the KP in microglia (Heyes et al., 1996) may contribute to neuronal and/or astrocytic cell death which may account for the reductions in brain volume 
which have been found in patients with chronic schizophrenia (van Erp et al., 2004). To this end, determining a role for KP activation in the pathophysiology of schizophrenia is complex as a consequence of inconsistent findings which may be due to the lack of standardised antipsychotic treatment in studies. Alongside this, physiological variations in patients may occur within different schizophrenic subgroups and between those experiencing acute or chronic schizophrenia.

A profile of astrocytic KP activation may account for raised circulating KYNA concentrations associated with the illness. A role for the KP and specifically KYNA in schizophrenia is further supported as NMDA receptor antagonists such as ketamine, phencyclidine, or MK-801 produce psychotomimetic effects in humans akin to the positive and cognitive symptoms characteristic of the illness (Radant et al., 1998). Systemically administered MK-801, which binds to the phencyclidine site of the NMDA receptor, produces social withdrawal and stimulates motor activity in rats, creating a model for positive and negative symptoms of schizophrenia, unlike amphetamine which provokes positive-related symptoms only (Rung et al., 2005). Furthermore Erhardt and Engberg (2002), have reported that increased KYNA concentrations, increase burst firing rate of midbrain dopaminergic neurons which is known to be involved in the pathophysiology of schizophrenia. This hyperactivity of dopaminergic neurons occurs in a similar fashion to responses obtained following MK-801, ketamine or phencyclidine administration (Erhardt and Engberg, 2002; Javitt and Zukin, 1991; Krystal et al., 1994), associating KYNA with the psychotomimetic properties of NMDA receptor antagonists.

With regards to peripherally produced KYNA, it may not necessarily play an important role in the pathophysiology of schizophrenia due to the inability of KYNA to cross the BBB. In this circumstance, pathological levels of KYNA may act at GPR35 mobilising intracellular calcium via inositol triphosphate production. Thus, pathological levels of central KYNA acting via neuronal GPR35 may initiate calcium-related excitotoxic and oxidative cascades independent of NMDA receptor activation. This may be a mechanism by which increased concentrations of KYNA in the brain may alter neuronal integrity and subsequently may contribute to behavioural symptoms displayed by schizophrenic patients although further investigation of this mechanism is required. It may be more so the production of peripheral kynurenine by hepatic TDO that is involved due to the ability of kynurenine to access the brain, providing astrocytes with a substrate from which KYNA can be produced. Schizophrenia is associated with disruption to the glutamatergic system which may be promoted as a result of KYNA-related antagonism of the NMDA receptor. This may in turn contribute to the array of behavioural symptoms observed in these patients. Koshy Cherian et al. (2014) reported that inhibition of KAT II may be beneficial in ameliorating some of the cognitive abnormalities experienced by schizophrenic patients. They reported that peripheral administration of the KAT II inhibitor, PF-04859989, to rats 30 min prior to kynurenine administration prevents increases in brain KYNA levels which was observed following administration of kynurenine. In addition, this group demonstrated that PF-04859989 restores nicotinicevoked glutamate release events which are otherwise attenuated by increased concentrations of KYNA in the brain. The authors suggest that this restoration occurs as a consequence of KYNA's antagonism at the $\alpha 7$-nicotinic receptor on glutamatergic thalamic afferents and/or the ionotropic glutamate receptors expressed by additional neuronal circuits which link stimulation of nicotinic receptors with glutamate release [see, Albuquerque and Schwarcz (2013) for review]. These glutamate release events were assessed on the basis of a previous finding where repeated pressure ejections of nicotine reliably stimulated glutamatergic release and thus, this may be a potential therapeutic mechanism for ameliorating disturbances in the glutamatergic system in schizophrenic patients.

Therapeutically, research has focused on developing KAT II inhibitors which interfere with the production of KYNA (Amaral et al., 2013). In addition, the TDO inhibitor, allopurinol, has been reported to show efficacy as adjuvant to standard antipsychotic treatment. Treatment with allopurinol was seen to non-significantly reduce the positive and negative syndrome scale, and patients receiving allopurinol rated themselves as more improved than those receiving placebo (Dickerson et al., 2009). Another recent case study has demonstrated that allopurinol was effective in improving the positive and negative symptoms of a patient who was otherwise unimproved for three months prior to beginning allopurinol adjuvant treatment (Linden et al., 2014).

\section{Targeting the KP; therapeutic considerations}

Considering the multi-step metabolism of tryptophan along the $\mathrm{KP}$, in addition to the branching of this pathway resulting in the production of neuroprotective or neurotoxic metabolites, there are multiple points where the pathway can be interrupted and modified. At present the competitive IDO inhibitor 1-methyl tryptophan is in clinical trials for the treatment of cancer (http:// clinicaltrials.gov/show/NCT01191216), while KMO and KAT II inhibitors are under investigation for the treatment of neurodegenerative and cognitive disorders respectively [see, Bohar et al. (2015), Dounay et al. (2015), Maddison and Giorgini (2015), Amaral et al. (2013) for review]. Thus far, TDO, IDO, KMO and KAT II exhibit the greatest potential as targets for drug development based on evidence emerging from both preclinical models and clinical studies which implicate a role for KP activation in CNS disorders.

\subsection{Targeting the rate limiting enzymes of the KP}

Allopurinol is now a widely accepted inhibitor of TDO, and renders enzymatic inhibition by way of preventing TDO from conjugating with its cofactor, heme (Green et al., 1976; Welch and Badawy, 1980). It is a purine analogue commonly used for treating gout due to its action as a xanthine oxidase inhibitor (Pacher et al., 2006). In addition, it has been repeatedly demonstrated that in the presence of stress, allopurinol is capable of reducing hepatic TDO activity (Green et al., 1976; Curzon and Green, 1969). The recently discovered TDO inhibitors, 680C91 and LM10 (Dolusic et al., 2011; Salter et al., 1995; Madge et al., 1996), show high selectivity for TDO over IDO (Pilotte et al., 2012), with LM10 showing chemotherapeutic efficacy in a pre-clinical tumour model in mice (Pilotte et al., 2012). It is suggested that simultaneous inhibition of both TDO and IDO may be a complementary approach rather than serving as conflicting pathways in cancer therapy (Pilotte et al., 2012). This combined approach could also be therapeutically relevant with regards to use in neuropsychiatric and degenerative disorders. Considering that these neurological disorders are linked with stress and exhibit an underlying inflammatory phenotype [see, Glass et al. (2010), Esch et al. (2002) for review], this dual-inhibitory approach may be efficacious as it would target both IDO- and TDO-driven KP activation. 1-Methyl tryptophan exists as either a dextro (D) or levo (L) enantiomer, with some in vivo and in vitro studies suggesting they have divergent actions. In vitro enzyme and cellular assays have demonstrated that the $L$ enantiomer is a more potent inhibitor of IDO (Qian et al., 2009; Lob et al., 2008), while in vivo studies have reported that the D enantiomer evokes a more effective anti-tumour response (Hou et al., 2007). The inconsistent findings are believed to be associated with the fact that the D enantiomer may have more off-target effects, and also that it is a more potent inhibitor of IDO-2 than IDO (Lob et al., 
2008). Other IDO inhibitors such as, imadizole, imadizothiazole, and triazole are extensively reviewed by Rohrig et al. (2015), and the apparent inconsistencies which occur with 1-methyl tryptophan also seem to appear with other current compounds. Considering that IDO is more so implicated in KP activation than IDO-2, further compounds have been developed including NLG919 which exhibits increased potency and ligand efficacy for IDO over IDO-2, in order to provide more effective experimental tools as well as potential novel therapeutic compounds [see, Dounay et al. (2015) for review].

\subsection{Targeting the KP downstream of TDO or IDO}

The first published KMO inhibitor, $m$-NBA, as well as other inhibitors including UPF648 and Ro61-8048 result in increased concentrations of plasma and brain KYNA and decreased central concentrations of 3-hydroxykynurenine when administered to rodents (Forrest et al., 2013a; Pellicciari et al., 2003; Chiarugi et al., 1996). JM6, originally believed to be a pro-drug of Ro61-8048 has been studied extensively and shows therapeutic potential in animal models of Huntington's and Alzheimer's disease [see, Bohar et al. (2015), Dounay et al. (2015), Amaral et al. (2013) for review]. To this end, the need for a BBB permeable KMO inhibitor has not been met. The development of such an agent would increase the potential for this enzyme to be a clinically relevant therapeutic target.

The current review highlights the varying role that KYNA has to play in both neurodegenerative and neuropsychiatric disorders. As such, it is not surprising that the transaminase class of KAT enzymes have become a potential therapeutic target in modulating the KP. Various KAT II inhibitors have been developed, including S-ESBA which showed selectivity for rat KAT II over KMO or KYNU, however it failed to inhibit the human orthologue (Rossi et al., 2010). A more promising inhibitor developed by Pfizer, PF-04859989, is irreversible and shows high selectivity for KAT II, the main driver of KYNA in the brain, over KAT I, III or IV isoforms in addition to it being capable of penetrating the BBB (Dounay et al., 2012). PF-04859989, will be a useful tool in deciphering the exact role that KYNA plays in various neurological disorders in terms of investigating the circumstances where it produces toxicity as opposed to its known protective function.

\subsection{Targeting the KP indirectly}

Considering that the neurotoxic enzymes IDO and KMO are induced by inflammatory stimuli both in vivo and in vitro (Connor et al., 2008) it is important to consider anti-inflammatory strategies as a method of manipulating KP activation. A study involving patients suffering from treatment resistant depression who received three infusions of the TNF $\alpha$ antagonist, infliximab indicated that this strategy although it is not effective in all circumstances, may improve depressive symptoms in patients with higher baseline inflammatory biomarkers (Raison et al., 2013). Furthermore, the non-steroidal anti-flammatory, diclofenac, which nonselectively inhibits cyclooxygenase (COX) -1 and -2 , increased the levels of rat brain KYNA following systemic administration. Similarly, indomethacin, which exhibits a slight preference for COX1 over COX-2, increased rat brain KYNA. In contrast, the COX-2 selective inhibitors parecoxib and meloxicam reduced the levels of central KYNA (Schwieler et al., 2005). Furthermore, $\beta$-adrenoceptor mediated anti-inflammatory effects may provide a novel mechanism of modulating the KP. The $\beta_{2}$-adrenoceptor agonist, clenbuterol was shown to have anti-inflammatory actions in rats following subcutaneous administration of kainic acid. Clenbuterol prevented the kainic acid-induced increases in inflammatory cytokines, IDO and KMO in the hippocampus of these rats (Gleeson et al., 2010). Moreover, various other $\beta$-agonists including noradrenaline, formoterol, and xamoterol, have been shown to increase KYNA production both in vitro and ex vivo in mixed glial cells and rat brain cortical slices respectively (Luchowska et al., 2009). Overall, modulation of the KP could be achieved through the use of various anti-inflammatory agents depending on the dysregulation of the pathway (see Muller (2013), for review). COX-2 selective inhibitors may be a beneficial adjunctive treatment in schizophrenia, while clenbuterol may be a more relevant adjunctive treatment in patients exhibiting an inflammatory phenotype and subsequently IDO activation. Similarly a study by Muller et al. (2010) which involved patients exhibiting their first manifestation of schizophrenia demonstrated celecoxib as an effective adjunctive treatment to amisulpride. This study showed that patients receiving both treatments had a more effective outcome in the positive and negative symptom scale and the clinical impression scale.

At present, there is scope for the development of compounds targeting other KP enzymes, such as KYNU, for which inhibitors do not already exist. In addition there is potential for modification of existing compounds in terms of BBB permeability and increased efficacy and selectivity. Considering the extent to which the KP and its downstream metabolites have been implicated in neurological disease, development of efficacious drugs which modulate this pathway either directly or indirectly may be beneficial clinically.

\section{Conclusions}

There is a role for stress-induced KP activation in neuropsychiatric and degenerative disorders, with the extent of its involvement varying between and within each disorder. The strongest evidence for KP dysregulation appears in major depressive disorder and schizophrenia, with over-activation of the pathway having a deleterious effect. Although inconsistencies exist, this may be as a consequence of the heterogeneity and co-morbid nature of mood disorders, alongside the variation in patient medications used, if any. The recognition that stress is associated with KP activation and a major contributor to both the pathophysiology and emergence of affective and neurodegenerative disorders places an emphasis on a likely role for stress-related regulation of the KP pathway in neurological disease. Although a role for inflammation-induced IDO in these disorders is proposed, a role for stress-induced TDO activation will require further investigation and development. Another interesting concept that to this end remains unexamined, is exploring how GR down-regulation, as a consequence of chronic stress may impact upon IDO expression in immune cells and/or TDO expression in the liver and brain. Investigation of this may further resolve the impact of KP activation and regulation in the pathophysiology of these disorders further clarifying if targeting the KP is a worthwhile pursuit therapeutically.

\section{Acknowledgements}

The authors would like to acknowledge grant support from the EUFP7 MOODINFLAME Consortium. Katherine O'Farrell is funded by a postgraduate award from Trinity College Dublin.

\section{References}

Agudelo, LZ, Femenia, T, Orhan, F, Porsmyr-Palmertz, M., Goiny, M., MartinezRedondo, V., Correia, J.C., Izadi, M., Bhat, M., Schuppe-Koistinen, I., Pettersson, A.T., Ferreira, D.M., Krook, A., Barres, R., Zierath, J.R., Erhardt, S. Lindskog, M., Ruas, J.L., 2014. Skeletal muscle PGC-1alpha1 modulates kynurenine metabolism and mediates resilience to stress-induced depression. Cell 159, 33-45.

Albelda, N., Joel, D., 2012. Animal models of obsessive-compulsive disorder: 
exploring pharmacology and neural substrates. Neurosci. Biobehav. Rev. 36, 47-63.

Alberati-Giani, D., Cesura, A.M., Broger, C., Warren, W.D., Röver, S., Malherbe, P. 1997. Cloning and functional expression of human kynurenine 3 monooxygenase. FEBS Lett. 410, 407-412.

Albuquerque, E.X., Schwarcz, R., 2013. Kynurenic acid as an antagonist of alpha7 nicotinic acetylcholine receptors in the brain: facts and challenges. Biochem. Pharmacol. 85, 1027-1032.

Amaral, M., Levy, C., Heyes, D.J., Lafite, P., Outeiro, T.F., Giorgini, F., Leys, D., Scrutton, N.S., 2013. Structural basis of kynurenine 3-monooxygenase inhibition. Nature 496, 382-385.

Anderson, G., Maes, M., 2014. TRYCAT pathways link peripheral inflammation, nicotine, somatization and depression in the etiology and course of Parkinson's disease. CNS Neurol. Disord. Drug Targets 13, 137-149.

Anderson, G., Rodriguez, M., 2011. Multiple sclerosis, seizures, and antiepileptics: role of IL-18, IDO, and melatonin. Eur. J. Neurol. 18, 680-685.

Asakura, M., Nagashima, H., Fujii, S., Sasuga, Y., Misonoh, A., Hasegawa, H., Osada, K., 2000. Influences of chronic stress on central nervous systems. Nihon Shinke Seishin Yakurigaku Zasshi 20, 97-105.

Ball, H.J., Sanchez-Perez, A., Weiser, S., Austin, C.J., Astelbauer, F., Miu, J., Mcquillan, J.A., Stocker, R., Jermiin, L.S., Hunt, N.H., 2007. Characterization of an indoleamine 2,3-dioxygenase-like protein found in humans and mice. Gene $396,203-213$.

Ball, H.J., Jusof, F.F., Bakmiwewa, S.M., Hunt, N.H., Yuasa, H.J., 2014. Tryptophancatabolizing enzymes - party of three. Front. Immunol. 5, 485.

Baran, H., Jellinger, K., Deecke, L., 1999. Kynurenine metabolism in Alzheimer's disease. J. Neural Transm. 106, 165-181.

Behan, W.M., Mcdonald, M., Darlington, L.G., Stone, T.W., 1999. Oxidative stress as a mechanism for quinolinic acid-induced hippocampal damage: protection by melatonin and deprenyl. Br. J. Pharmacol. 128, 1754-1760.

Berger, S.J., Sudar, D.C., Berger, N.A., 1986. Metabolic consequences of DNA damage: DNA damage induces alterations in glucose metabolism by activation of poly (ADP-ribose) polymerase. Biochem. Biophys. Res. Commun. 134, 227-232.

Berger, F., Ramirez-Hernandez, M.H., Ziegler, M., 2004. The new life of a centenarian: signalling functions of NAD(P). Trends Biochem. Sci. 29, 111-118.

Birch, P.J., Grossman, C.J., Hayes, A.G., 1988a. Kynurenate and FG9041 have both competitive and non-competitive antagonist actions at excitatory amino acid receptors. Eur. J. Pharmacol. 151, 313-315.

Birch, P.J., Grossman, C.J., Hayes, A.G., 1988b. Kynurenic acid antagonises responses to NMDA via an action at the strychnine-insensitive glycine receptor. Eur. J. Pharmacol. 154, 85-87.

Bohar, Z., Toldi, J., Fulop, F., Vecsei, L., 2015. Changing the face of kynurenines and neurotoxicity: therapeutic considerations. Int. J. Mol. Sci. 16, 9772-9793.

Bozza, S., Fallarino, F., Pitzurra, L., Zelante, T., Montagnoli, C., Bellocchio, S., Mosci, P. Vacca, C., Puccetti, P., Romani, L., 2005. A crucial role for tryptophan catabolism at the host/Candida albicans interface. J. Immunol. 174, 2910-2918.

Braidy, N., Grant, R., Adams, S., Brew, B.J., Guillemin, G.J., 2009a. Mechanism for quinolinic acid cytotoxicity in human astrocytes and neurons. Neurotox. Res. 16 77-86.

Braidy, N., Grant, R., Brew, B.J., Adams, S., Jayasena, T., Guillemin, G.J., 2009b. Effects of kynurenine pathway metabolites on intracellular NAD synthesis and cel death in human primary astrocytes and neurons. Int. J. Tryptophan Res. 2, $61-69$

Bronte, V., Pittet, M.J., 2013. The spleen in local and systemic regulation of immunity. Immunity 39, 806-818

Cazzullo, C.L., Sacchetti, E., Galluzzo, A., Panariello, A., Adorni, A., Pegoraro, M. Bosis, S., Colombo, F., Trabattoni, D., Zagliani, A., Clerici, M., 2002. Cytokine profiles in schizophrenic patients treated with risperidone: a 3-month followup study. Prog. Neuropsychopharmacol. Biol. Psychiatry 26, 33-39.

Chen, Y., Guillemin, G.J., 2009. Kynurenine pathway metabolites in humans: disease and healthy States. Int. J. Tryptophan Res. 2, 1-19.

Chen, Y. \& Guillemin, G. 2012. The kynurenine pathway. In: Maure, M. (ed.). InTech.

Chen, Y., Brew, B.J., Guillemin, G.J., 2011. Characterization of the kynurenine pathway in NSC-34 cell line: implications for amyotrophic lateral sclerosis. J. Neurochem 118, 816-825.

Chiappelli, J., Pocivavsek, A., Nugent, K.L., Notarangelo, F.M., Kochunov, P., Rowland, L.M., Schwarcz, R., Hong, L.E., 2014. Stress-induced increase in kynurenic acid as a potential biomarker for patients with schizophrenia and distress intolerance. JAMA Psychiatry 71, 761-768.

Chiarugi, A., Carpenedo, R., Moroni, F., 1996. Kynurenine disposition in blood and brain of mice: effects of selective inhibitors of kynurenine hydroxylase and of kynureninase. J. Neurochem. 67, 692-698.

Chiarugi, A., Dello Sbarba, P., Paccagnini, A., Donnini, S., Filippi, S., Moroni, F., 2000 Combined inhibition of indoleamine 2,3-dioxygenase and nitric oxide synthase modulates neurotoxin release by interferon-gamma-activated macrophages. J. Leukoc. Biol. 68, 260-266.

Chiarugi, A., Cozzi, A., Ballerini, C., Massacesi, L., Moroni, F., 2001. Kynurenine 3 mono-oxygenase activity and neurotoxic kynurenine metabolites increase in the spinal cord of rats with experimental allergic encephalomyelitis. Neuroscience 102, 687-695.

Chiba, S., Numakawa, T., Ninomiya, M., Richards, M.C., Wakabayashi, C., Kunugi, H. 2012. Chronic restraint stress causes anxiety- and depression-like behaviors, downregulates glucocorticoid receptor expression, and attenuates glutamate release induced by brain-derived neurotrophic factor in the prefrontal cortex. Prog. Neuropsychopharmacol. Biol. Psychiatry 39, 112-119.
Comings, D.E., 2001. Clinical and molecular genetics of ADHD and Tourette syndrome. Two related polygenic disorders. Ann. N. Y. Acad. Sci. 931, 50-83.

Connor, T.J., Starr, N., O'Sullivan, J.B., Harkin, A., 2008. Induction of indolamine 2,3dioxygenase and kynurenine 3-monooxygenase in rat brain following a systemic inflammatory challenge: a role for IFN-gamma? Neurosci. Lett. 441, $29-34$.

Cuartero, M.I., Ballesteros, I., de la Parra, J., Harkin, A.L., Abautret-Daly, A., Sherwin, E., Fernandez-Salguero, P., Corbi, A.L., Lizasoain, I., Moro, M.A., 2014. Lkynurenine/aryl hydrocarbon receptor pathway mediates brain damage after experimental stroke. Circulation 130, 2040-2051.

Curzon, G., Green, A.R., 1969. Effects of immobilization on rat liver tryptophan pyrrolase and brain 5-hydroxytryptamine metabolism. Br. J. Pharmacol. 37, 689-697.

Cutler, J.A., Rush, A.J., Mcmahon, F.J., Laje, G., 2012. Common genetic variation in the indoleamine-2,3-dioxygenase genes and antidepressant treatment outcome in major depressive disorder. J. Psychopharmacol. 26, 360-367.

Darlington, L.G., Mackay, G.M., Forrest, C.M., Stoy, N., George, C., Stone, T.W., 2007. Altered kynurenine metabolism correlates with infarct volume in stroke. Eur. J. Neurosci. 26, 2211-2221.

Deacon, R.M., 2013. The successive alleys test of anxiety in mice and rats. J. Vis. Exp. (76)

Dickerson, F.B., Stallings, C.R., Origoni, A.E., Sullens, A., Khushalani, S., Sandson, N., Yolken, R.H., 2009. A double-blind trial of adjunctive allopurinol for schizophrenia. Schizophr. Res. 109, 66-69.

Divorty, N., Mackenzie, A.E., Nicklin, S.A., Milligan, G., 2015. G protein-coupled receptor 35: an emerging target in inflammatory and cardiovascular disease. Front. Pharmacol, 6, 41

Dolusic, E., Larrieu, P., Moineaux, L., Stroobant, V., Pilotte, L., Colau, D., Pochet, L., Van den Eynde, B., Masereel, B., Wouters, J., Frederick, R., 2011. Tryptophan 2,3dioxygenase (TDO) inhibitors. 3-(2-(pyridyl)ethenyl)indoles as potential anticancer immunomodulators. J. Med. Chem. 54, 5320-5334.

Dong, Y., Poellinger, L., Gustafsson, J.A., Okret, S., 1988. Regulation of glucocorticoid receptor expression: evidence for transcriptional and posttranslational mechanisms. Mol. Endocrinol. 2, 1256-1264.

Dong-Ryul, L., Kondo, H., Furukawa, S., Nakano, K., 1997. Stimulation of NGF production by tryptophan and its metabolites in cultured mouse astroglial cells. Brain Res. 777, 228-230.

Dounay, A.B., Anderson, M., Bechle, B.M., Campbell, B.M., Claffey, M.M., Evdokimov, A., Evrard, E., Fonseca, K.R., Gan, X., Ghosh, S., Hayward, M.M., Horner, W., Kim, J.Y., Mcallister, L.A., Pandit, J., Paradis, V., Parikh, V.D. Reese, M.R., Rong, S., Salafia, M.A., Schuyten, K., Strick, C.A., Tuttle, J.B. Valentine, J., Wang, H., Zawadzke, L.E., Verhoest, P.R., 2012. Discovery of brainpenetrant, irreversible kynurenine aminotransferase II inhibitors for schizophrenia. ACS Med. Chem. Lett. 3, 187-192.

Dounay, A.B., Tuttle, J.B., Verhoest, P.R., 2015. Challenges and opportunities in the discovery of new therapeutics targeting the kynurenine pathway. J. Med. Chem. 58 (22), 8762-8782.

Dunn, A.J. Swiergiel, A.H., de Beaurepaire, R., 2005. Cytokines as mediators of depression: what can we learn from animal studies? Neurosci. Biobehav. Rev. 29, 891-909.

Elenkov, I.J., Wilder, R.L., Chrousos, G.P., Vizi, E.S., 2000. The sympathetic nerve-an integrative interface between two supersystems: the brain and the immune system. Pharmacol. Rev. 52, 595-638.

Erhardt, S., Engberg, G., 2002. Increased phasic activity of dopaminergic neurones in the rat ventral tegmental area following pharmacologically elevated levels of endogenous kynurenic acid. Acta Physiol. Scand. 175, 45-53.

Erickson, J.B., Flanagan, E.M., Russo, S., Reinhard Jr., J.F., 1992. A radiometric assay for kynurenine 3-hydroxylase based on the release of $3 \mathrm{H}_{2} \mathrm{O}$ during hydroxylation of L-[3,5-3H]kynurenine. Anal. Biochem. 205, 257-262.

Esch, T., Stefano, G.B., Fricchione, G.L., Benson, H., 2002. The role of stress in neurodegenerative diseases and mental disorders. Neuro Endocrinol. Lett. 23, 199-208.

Fallarino, F., Grohmann, U., Vacca, C., Bianchi, R., Orabona, C., Spreca, A., Fioretti, M.C., Puccetti, P., 2002. T cell apoptosis by tryptophan catabolism. Cell Death Differ. 9, 1069-1077.

Faravelli, C., Lo Sauro, C., Godini, L., Lelli, L., Benni, L., Pietrini, F., Lazzeretti, L. Talamba, G.A., Fioravanti, G., Ricca, V., 2012. Childhood stressful events, HPA axis and anxiety disorders. World J. Psychiatry 2, 13-25.

Fatokun, A.A., Hunt, N.H., Ball, H.J., 2013. Indoleamine 2,3-dioxygenase 2 (IDO2) and the kynurenine pathway: characteristics and potential roles in health and disease. Amino Acids 45, 1319-1329.

Federico, A., Cardaioli, E., Da Pozzo, P., Formichi, P., Gallus, G.N., Radi, E., 2012 Mitochondria, oxidative stress and neurodegeneration. J. Neurol. Sci. 322, 254-262.

Ferrante, M., Migliore, M., Ascoli, G.A., 2013. Functional impact of dendritic branchpoint morphology. J. Neurosci. 33, 2156-2165.

Forouhar, F., Anderson, J.L., Mowat, C.G., Vorobiev, S.M., Hussain, A., Abashidze, M., Bruckmann, C., Thackray, S.J., Seetharaman, J., Tucker, T., Xiao, R., Ma, L.C., Zhao, L., Acton, T.B., Montelione, G.T., Chapman, S.K., Tong, L., 2007. Molecular insights into substrate recognition and catalysis by tryptophan 2,3-dioxygenase. Proc. Natl. Acad. Sci. U. S. A. 104, 473-478.

Forrest, C.M., Mackay, G.M., Stoy, N., Egerton, M., Christofides, J., Stone, T.W., Darlington, L.G. 2004. Tryptophan loading induces oxidative stress. Free Radic. Res. 38, 1167-1171.

Forrest, C.M., Khalil, O.S., Pisar, M., Darlington, L.G., Stone, T.W., 2013a. Prenatal 
inhibition of the tryptophan-kynurenine pathway alters synaptic plasticity and protein expression in the rat hippocampus. Brain Res. 1504, 1-15.

Forrest, C.M., Khalil, O.S., Pisar, M., Mcnair, K., Kornisiuk, E., Snitcofsky, M., Gonzalez, N., Jerusalinsky, D., Darlington, L.G., Stone, T.W., 2013b. Changes in synaptic transmission and protein expression in the brains of adult offspring after prenatal inhibition of the kynurenine pathway. Neuroscience 254, 241-259.

Fukuda, K., 2014. Etiological classification of depression based on the enzymes of tryptophan metabolism. BMC Psychiatry 14, 372.

Fukui, S., Schwarcz, R., Rapoport, S.I., Takada, Y., Smith, Q.R., 1991. Blood-brain barrier transport of kynurenines: implications for brain synthesis and metabolism. J. Neurochem. 56, 2007-2017.

Fukunaga, M., Yamamoto, Y., Kawasoe, M., Arioka, Y., Murakami, Y., Hoshi, M. Saito, K., 2012. Studies on tissue and cellular distribution of indoleamine 2,3dioxygenase 2: the absence of IDO1 upregulates IDO2 expression in the epididymis. J. Histochem. Cytochem. 60, 854-860.

Funakoshi, Masaaki, K., Toshikazu, N., 2011a. Modulation of tryptophan metabolism, promotion of neurogenesis and alteration of anxiety-related behavior in tryptophan 2,3-dioxygenase-deficient mice. Int. J. Tryptophan Res. 7.

Funakoshi, H., Kanai, M., Nakamura, T., 2011b. Modulation of tryptophan metabolism, pomotion of neurogenesis and alteration of anxiety-related behavior in tryptophan 2,3-dioxygenase-deficient mice. Int. J. Tryptophan Res. IJTR 4, 7-18.

Furtado, M., Katzman, M.A., 2015. Neuroinflammatory pathways in anxiety, posttraumatic stress, and obsessive compulsive disorders. Psychiatry Res. 229, $37-48$.

Gallowitsch-Puerta, M., Tracey, K.J., 2005. Immunologic role of the cholinergic antiinflammatory pathway and the nicotinic acetylcholine alpha 7 receptor. Ann. N. Y. Acad. Sci. 1062, 209-219.

Gaspar, P., Cases, O., Maroteaux, L., 2003. The developmental role of serotonin: news from mouse molecular genetics. Nat. Rev. Neurosci. 4, 1002-1012.

Gibney, S.M., Mcguinness, B., Prendergast, C., Harkin, A., Connor, T.J., 2013. Poly I: Cinduced activation of the immune response is accompanied by depression and anxiety-like behaviours, kynurenine pathway activation and reduced BDNF expression. Brain Behav. Immun. 28, 170-181.

Gibney, S.M., Fagan, E.M., Waldron, A.M., O'Byrne, J., Connor, T.J., Harkin, A., 2014. Inhibition of stress-induced hepatic tryptophan 2,3-dioxygenase exhibits antidepressant activity in an animal model of depressive behaviour. Int. J. Neuropsychopharmacol. 17, 917-928.

Gigler, G., Szenasi, G., Simo, A., Levay, G., Harsing Jr., L.G., Sas, K., Vecsei, L., Toldi, J., 2007. Neuroprotective effect of L-kynurenine sulfate administered before focal cerebral ischemia in mice and global cerebral ischemia in gerbils. Eur. J. Pharmacol. 564, 116-122.

Glass, C.K., Saijo, K., Winner, B., Marchetto, M.C., Gage, F.H., 2010. Mechanisms underlying inflammation in neurodegeneration. Cell 140, 918-934.

Gleeson, L.C., Ryan, K.J., Griffin, E.W., Connor, T.J., Harkin, A., 2010. The beta2adrenoceptor agonist clenbuterol elicits neuroprotective, anti-inflammatory and neurotrophic actions in the kainic acid model of excitotoxicity. Brain Behav. Immun. 24, 1354-1361.

Goswami, S., Rodriguez-Sierra, O., Cascardi, M., Pare, D., 2013. Animal models of post-traumatic stress disorder: face validity. Front. Neurosci. 7, 89.

Gracia-Rubio, I., Moscoso-Castro, M., Pozo, O.J., Marcos, J., Nadal, R., Valverde, O., 2015. Maternal separation induces neuroinflammation and long-lasting emotional alterations in mice. Prog. Neuropsychopharmacol. Biol. Psychiatry 65, 104-117.

Gray, S.M., Bloch, M.H., 2012. Systematic review of proinflammatory cytokines in obsessive-compulsive disorder. Curr. Psychiatry Rep. 14, 220-228.

Green, A.R., Curzon, G., 1968. Decrease of 5-hydroxytryptamine in the brain provoked by hydrocortisone and its prevention by allopurinol. Nature 220, 1095-1097.

Green, A.R., Curzon, G., 1975. Effects of hydrocortisone and immobilization on tryptophan metabolism in brain and liver of rats of different ages. Biochem. Pharmacol. 24, 713-716.

Green, A.R., Woods, H.F., Joseph, M.H., 1976. Tryptophan metabolism in the isolated perfused liver of the rat: effects of tryptophan concentration, hydrocortisone and allopurinol on tryptophan pyrrolase activity and kynurenine formation. $\mathrm{Br}$ J. Pharmacol. 57, 103-114.

Gregoire, L., Rassoulpour, A., Guidetti, P., Samadi, P., Bedard, P.J., Izzo, E., Schwarcz, R., Di Paolo, T., 2008. Prolonged kynurenine 3-hydroxylase inhibition reduces development of levodopa-induced dyskinesias in parkinsonian monkeys. Behav. Brain Res. 186, 161-167.

Guidetti, P., Schwarcz, R., 2003. 3-Hydroxykynurenine and quinolinate: pathogenic synergism in early grade Huntington's disease? Adv. Exp. Med. Biol. 527, 137-145.

Guidetti, P., Reddy, P.H., Tagle, D.A., Schwarcz, R., 2000. Early kynurenergic impairment in Huntington's disease and in a transgenic animal model. Neurosci. Lett. 283, 233-235.

Guidetti, P., Luthi-Carter, R.E., Augood, S.J., Schwarcz, R., 2004. Neostriatal and cortical quinolinate levels are increased in early grade Huntington's disease. Neurobiol. Dis, 17, 455-461.

Guillemin, G.J., 2012. Quinolinic acid, the inescapable neurotoxin. FEBS J. 279, 1356-1365.

Guillemin, G.J., Brew, B.J., 2002. Implications of the kynurenine pathway and quinolinic acid in Alzheimer's disease. Redox Rep. 7, 199-206.

Guillemin, G.J., Kerr, S.J., Smythe, G.A., Smith, D.G., Kapoor, V., Armati, P.J., Croitoru, J., Brew, B.J., 2001. Kynurenine pathway metabolism in human astrocytes: a paradox for neuronal protection. J. Neurochem. 78, 842-853.

Guillemin, G.J., Smith, D.G., Smythe, G.A., Armati, P.J., Brew, B.J., 2003a. Expression of the kynurenine pathway enzymes in human microglia and macrophages. Adv. Exp. Med. Biol. 527, 105-112.

Guillemin, G.J., Smythe, G.A., Veas, L.A., Takikawa, O., Brew, B.J., 2003b. A beta 1-42 induces production of quinolinic acid by human macrophages and microglia. Neuroreport 14, 2311-2315.

Guillemin, G.J., Kerr, S.J., Brew, B.J., 2005a. Involvement of quinolinic acid in AIDS dementia complex. Neurotox. Res. 7, 103-123.

Guillemin, G.J., Smythe, G., Takikawa, O., Brew, B.J., 2005b. Expression of indoleamine 2,3-dioxygenase and production of quinolinic acid by human microglia, astrocytes, and neurons. Glia 49, 15-23.

Guillemin, G.J., Cullen, K.M., Lim, C.K., Smythe, G.A., Garner, B., Kapoor, V. Takikawa, O., Brew, B.J., 2007. Characterization of the kynurenine pathway in human neurons. J. Neurosci. 27, 12884-12892.

Haroon, E., Raison, C.L., Miller, A.H., 2012. Psychoneuroimmunology meets neuropsychopharmacology: translational implications of the impact of inflammation on behavior. Neuropsychopharmacology 37, 137-162.

Heisler, J.M., O'Connor, J.C., 2015. Indoleamine 2,3-dioxygenase-dependent neurotoxic kynurenine metabolism mediates inflammation-induced deficit in recognition memory. Brain Behav. Immun. 50, 115-124.

Heyes, M.P., Achim, C.L., Wiley, C.A., Major, E.O., Saito, K., Markey, S.P., 1996. Human microglia convert l-tryptophan into the neurotoxin quinolinic acid. Biochem. J. 320 (Pt 2), 595-597.

Hill, M., Tanguy-Royer, S., Royer, P., Chauveau, C., Asghar, K., Tesson, L., Lavainne, F. Remy, S., Brion, R., Hubert, F.X., Heslan, M., Rimbert, M., Berthelot, L. Moffett, J.R., Josien, R., Gregoire, M., Anegon, I., 2007. IDO expands human $\mathrm{CD} 4+\mathrm{CD} 25$ high regulatory $\mathrm{T}$ cells by promoting maturation of LPS-treated dendritic cells. Eur. J. Immunol. 37, 3054-3062.

Hilmas, C., Pereira, E.F., Alkondon, M., Rassoulpour, A., Schwarcz, R., Albuquerque, E.X., 2001. The brain metabolite kynurenic acid inhibits alpha7 nicotinic receptor activity and increases non-alpha7 nicotinic receptor expression: physiopathological implications. J. Neurosci. 21, 7463-7473.

Holtzman, C.W., Trotman, H.D., Goulding, S.M., Ryan, A.T., Macdonald, A.N Shapiro, D.I., Brasfield, J.L., Walker, E.F., 2013. Stress and neurodevelopmental processes in the emergence of psychosis. Neuroscience 249, 172-191.

Hou, D.Y., Muller, A.J., Sharma, M.D., Duhadaway, J., Banerjee, T., Johnson, M. Mellor, A.L., Prendergast, G.C., Munn, D.H., 2007. Inhibition of indoleamine 2,3 dioxygenase in dendritic cells by stereoisomers of 1-methyl-tryptophan correlates with antitumor responses. Cancer Res. 67, 792-801.

Hwu, P. Du, M.X. Lapointe, R., Do, M. Taylor, M.W. Young, H.A., 2000. Indoleamine 2,3-dioxygenase production by human dendritic cells results in the inhibition of T cell proliferation. J. Immunol. 164, 3596-3599.

Javitt, D.C., Zukin, S.R., 1991. Recent advances in the phencyclidine model of schizophrenia. Am. J. Psychiatry 148, 1301-1308.

Jenkins, L., Alvarez-Curto, E., Campbell, K., de Munnik, S., Canals, M., Schlyer, S. Milligan, G., 2011. Agonist activation of the G protein-coupled receptor GPR35 involves transmembrane domain III and is transduced via Galpha(1)(3) and beta-arrestin-2. Br. J. Pharmacol. 162, 733-748.

Johnson, P.L., Shekhar, A., 2012. An animal model of panic vulnerability with chronic disinhibition of the dorsomedial/perifornical hypothalamus. Physiol. Behav. 107, 686-698.

Jones, S.P., Franco, N.F., Varney, B., Sundaram, G., Brown, D.A., de Bie, J., Lim, C.K. Guillemin, G.J., Brew, B.J., 2015. Expression of the kynurenine pathway in human peripheral blood mononuclear cells: implications for inflammatory and neurodegenerative disease. PLoS One 10, e0131389.

Kanai, M., Funakoshi, H., Takahashi, H., Hayakawa, T., Mizuno, S., Matsumoto, K, Nakamura, T., 2009a. Tryptophan 2,3-dioxygenase is a key modulator of physiological neurogenesis and anxiety-related behavior in mice. Mol. Brain 2, 8 .

Kanai, M., Nakamura, T., Funakoshi, H., 2009b. Identification and characterization of novel variants of the tryptophan 2,3-dioxygenase gene: differential regulation in the mouse nervous system during development. Neurosci. Res. 64, 111-117.

Kanai, M., Funakoshi, H., Nakamura, T., 2010. Implication of tryptophan 2,3dioxygenase and its novel variants in the hippocampus and cerebellum during the developing and adult brain. Int. J. Tryptophan Res. IJTR 3, 141-149.

Karakuła-Juchnowicz, H., Flis, M., Szymona, K., Kuczyńska, M., Stelmach, E., KowalPopczak, A., 2015. New prospects for antipsychotic treatment - the role of the kynurenine pathway. Psychiatry Pol. 48, 1167-1177.

Kerr, S.J. Armati, P.J. Guillemin, G.J. Brew, B.J. 1998. Chronic exposure of human neurons to quinolinic acid results in neuronal changes consistent with AIDS dementia complex. Aids 12, 355-363.

Khalil, O.S., Pisar, M., Forrest, C.M., Vincenten, M.C.J., Darlington, L.G., Stone, T.W. 2014. Prenatal inhibition of the kynurenine pathway leads to structural changes in the hippocampus of adult rat offspring. Eur. J. Neurosci. 39, 1558-1571.

Kim, Y.K., Myint, A.M., Verkerk, R., Scharpe, S., Steinbusch, H., Leonard, B., 2009 Cytokine changes and tryptophan metabolites in medication-naive and medication-free schizophrenic patients. Neuropsychobiology 59, 123-129.

Kim, J., Hong, H., Heo, A., Park, W., 2013. Indole toxicity involves the inhibition of adenosine triphosphate production and protein folding in Pseudomonas putida. FEMS Microbiol. Lett. 343, 89-99.

Knyihar-Csillik, E., Csillik, B., Pakaski, M., Krisztin-Peva, B., Dobo, E., Okuno, E. Vecsei, L., 2004. Decreased expression of kynurenine aminotransferase-I (KAT-I) in the substantia nigra of mice after 1-methyl-4-phenyl-1,2,3,6tetrahydropyridine (MPTP) treatment. Neuroscience 126, 899-914.

Kocki, T., Luchowski, P., Luchowska, E., Wielosz, M., Turski, W.A., Urbanska, E.M., 
2003. L-cysteine sulphinate, endogenous sulphur-containing amino acid, inhibits rat brain kynurenic acid production via selective interference with kynurenine aminotransferase II. Neurosci. Lett. 346, 97-100.

Koenig, J.I., Kirkpatrick, B., Lee, P., 2002. Glucocorticoid hormones and early brain development in schizophrenia. Neuropsychopharmacology 27, 309-318.

Kohm, A.P., Sanders, V.M., 2001. Norepinephrine and beta 2-adrenergic receptor stimulation regulate $\mathrm{CD} 4+\mathrm{T}$ and $\mathrm{B}$ lymphocyte function in vitro and in vivo. Pharmacol. Rev. 53, 487-525.

Koshy Cherian, A., Gritton, H., Johnson, D.E., Young, D., Kozak, R., Sarter, M., 2014 A systemically-available kynurenine aminotransferase II (KAT II) inhibitor restores nicotine-evoked glutamatergic activity in the cortex of rats. Neuropharmacology $82,41-48$.

Krystal, J.H., Karper, L.P., Seibyl, J.P., Freeman, G.K., Delaney, R., Bremner, J.D., Heninger, G.R., Bowers Jr., M.B., Charney, D.S., 1994. Subanesthetic effects of the noncompetitive NMDA antagonist, ketamine, in humans. Psychotomimetic perceptual, cognitive, and neuroendocrine responses. Arch. Gen. Psychiatry 51, 199-214.

Kubesova, A., Tejkalova, H., Syslova, K., Kacer, P., Vondrousova, J., Tyls, F. Fujakova, M., Palenicek, T., Horacek, J., 2015. Biochemical, histopathological and morphological profiling of a rat model of early immune stimulation: relation to psychopathology. PLoS One 10, e0115439.

Kwidzinski, E., Bechmann, I., 2007. IDO expression in the brain: a double-edged sword. J. Mol. Med. (Berl.) 85, 1351-1359.

Lauder, J.M., 1990. Ontogeny of the serotonergic system in the rat: serotonin as developmental signal. Ann. N. Y. Acad. Sci. 600, 297-313 discussion 314.

Laugeray, A., Launay, J.M., Callebert, J., Surget, A., Belzung, C., Barone, P.R., 2011. Evidence for a key role of the peripheral kynurenine pathway in the modulation of anxiety- and depression-like behaviours in mice: focus on individual differences. Pharmacol. Biochem. Behav. 98, 161-168.

Lawson, M.A., Parrott, J.M., Mccusker, R.H., Dantzer, R., Kelley, K.W., O'Connor, J.C., 2013. Intracerebroventricular administration of lipopolysaccharide induces indoleamine-2,3-dioxygenase-dependent depression-like behaviors. J. Neuroinflamm. 10, 87.

Lim, C.K., Brew, B.J., Sundaram, G. Guillemin, G.J., 2010. Understanding the roles of the kynurenine pathway in multiple sclerosis progression. Int. J. Tryptophan Res. 3, 157-167.

Linden, N., Onwuanibe, A., Sandson, N., 2014. Rapid resolution of psychotic symptoms in a patient with schizophrenia using allopurinol as an adjuvant: a case report. Clin. Schizophr. Relat. Psychoses 7, 231-234.

Lob, S., Konigsrainer, A., Schafer, R., Rammensee, H.G., Opelz, G., Terness, P., 2008. Levo- but not dextro-1-methyl tryptophan abrogates the IDO activity of human dendritic cells. Blood 111, 2152-2154.

Lopresti, A.L., Maker, G.L., Hood, S.D., Drummond, P.D., 2014. A review of periphera biomarkers in major depression: the potential of inflammatory and oxidative stress biomarkers. Prog. Neuropsychopharmacol. Biol. Psychiatry 48, 102-111.

Lucassen, P.J., Naninck, E.F., van Goudoever, J.B., Fitzsimons, C., Joels, M., Korosi, A 2013. Perinatal programming of adult hippocampal structure and function; merging roles of stress, nutrition and epigenetics. Trends Neurosci. 36, $621-631$.

Luchowska, E., Kloc, R., Olajossy, B., Wnuk, S., Wielosz, M., Owe-Larsson, B. Urbanska, E.M., 2009. beta-adrenergic enhancement of brain kynurenic acid production mediated via cAMP-related protein kinase A signaling. Prog. Neuropsychopharmacol. Biol. Psychiatry 33, 519-529.

Luchowski, P., Luchowska, E., Turski, W.A., Urbanska, E.M., 2002. 1-Methyl-4phenylpyridinium and 3-nitropropionic acid diminish cortical synthesis of kynurenic acid via interference with kynurenine aminotransferases in rats. Neurosci. Lett. 330, 49-52.

Lugo-Huitron, R., Ugalde Muniz, P., Pineda, B., Pedraza-Chaverri, J., Rios, C., Perez-de la Cruz, V., 2013. Quinolinic acid: an endogenous neurotoxin with multiple targets. Oxid. Med. Cell. Longev. 2013, 104024.

Maddison, D.C., Giorgini, F., 2015. The kynurenine pathway and neurodegenerative disease Semin Cell Dev. Biol. 40, 134-141.

Madge, D., Hazelwood, R., Iyer, R., Jones, H., Salter, M., 1996. Novel tryptophan dioxygenase inhibitors and combined tryptophan dioxygenase/5-HT reuptake inhibitors. Bioorg. Med. Chem. Lett. 6, 857-860.

Maes, M., 2011. Depression is an inflammatory disease, but cell-mediated immune activation is the key component of depression. Prog. Neuropsychopharmacol. Biol. Psychiatry 35, 664-675.

Maes, M., Verkerk, R., Bonaccorso, S., Ombelet, W., Bosmans, E., Scharpe, S., 2002 Depressive and anxiety symptoms in the early puerperium are related to increased degradation of tryptophan into kynurenine, a phenomenon which is related to immune activation. Life Sci. 71, 1837-1848.

Maes, M., Leonard, B.E., Myint, A.M., Kubera, M., Verkerk, R., 2011. The new '5-HT' hypothesis of depression: cell-mediated immune activation induces indole amine 2,3-dioxygenase, which leads to lower plasma tryptophan and an increased synthesis of detrimental tryptophan catabolites (TRYCATs), both of which contribute to the onset of depression. Prog. Neuropsychopharmacol. Biol. Psychiatry 35, 702-721.

Maisel, A.S., Fowler, P., Rearden, A., Motulsky, H.J., Michel, M.C., 1989. A new method for isolation of human lymphocyte subsets reveals differential regulation of beta-adrenergic receptors by terbutaline treatment. Clin. Pharmacol. Ther. 46, 429-439.

Marchi, M. Risso, F., Viola, C., Cavazzani, P., Raiteri, M., 2002. Direct evidence that release-stimulating alpha7* nicotinic cholinergic receptors are localized on human and rat brain glutamatergic axon terminals. J. Neurochem. 80
$1071-1078$

Marques, A.H., Silverman, M.N., Sternberg, E.M., 2009. Glucocorticoid dysregulations and their clinical correlates. From receptors to therapeutics. Ann. N. Y. Acad. Sci. 1179, 1-18.

Marti-Masso, J.F., Bergareche, A., Makarov, V., Ruiz-Martinez, J., Gorostidi, A., Lopez de Munain, A., Poza, J.J., Striano, P., Buxbaum, J.D., Paisan-Ruiz, C., 2013. The ACMSD gene, involved in tryptophan metabolism, is mutated in a family with cortical myoclonus, epilepsy, and parkinsonism. J. Mol. Med. (Berl.) 91, 1399-1406.

Martin, D., Thompson, M.A., Nadler, J.V., 1993. The neuroprotective agent riluzole inhibits release of glutamate and aspartate from slices of hippocampal area CA1. Eur. J. Pharmacol. 250, 473-476.

McEwen, B.S., 2008. Understanding the potency of stressful early life experiences on brain and body function. Metab. Clin. Exp. 57, S11-S15.

Miller, C.L., Llenos, I.C., Dulay, J.R., Barillo, M.M., Yolken, R.H., Weis, S., 2004. Expression of the kynurenine pathway enzyme tryptophan 2,3-dioxygenase is increased in the frontal cortex of individuals with schizophrenia. Neurobiol. Dis. $15,618-629$.

Miller, G.E., Chen, E., Sze, J., Marin, T., Arevalo, J.M., Doll, R., Ma, R., Cole, S.W., 2008. A functional genomic fingerprint of chronic stress in humans: blunted glucocorticoid and increased NF-kappaB signaling. Biol. Psychiatry 64, 266-272.

Miranda, A.F., Boegman, R.J., Beninger, R.J., Jhamandas, K., 1997. Protection against quinolinic acid-mediated excitotoxicity in nigrostriatal dopaminergic neurons by endogenous kynurenic acid. Neuroscience 78, 967-975.

Moroni, F., Russi, P., Lombardi, G., Beni, M., Carla, V., 1988. Presence of kynurenic acid in the mammalian brain. J. Neurochem. 51, 177-180.

Muller, N., 2013. The role of anti-inflammatory treatment in psychiatric disorders. Psychiatr. Danub. 25, 292-298.

Muller, N., Schwarz, M.J., 2010. Immune system and schizophrenia. Curr. Immunol. Rev. 6, 213-220.

Muller, N., Krause, D., Dehning, S., Musil, R., Schennach-Wolff, R., Obermeier, M., Moller, H.J., Klauss, V., Schwarz, M.J., Riedel, M., 2010. Celecoxib treatment in an early stage of schizophrenia: results of a randomized, double-blind, placebocontrolled trial of celecoxib augmentation of amisulpride treatment. Schizophr. Res. 121, 118-124.

Munn, D.H., 2011. Indoleamine 2,3-dioxygenase, Tregs and cancer. Curr. Med. Chem. 18, 2240-2246.

Myint, A.M., Kim, Y.K., 2003. Cytokine-serotonin interaction through IDO: a neurodegeneration hypothesis of depression. Med. Hypotheses 61, 519-525.

Myint, A.M., Kim, Y.K., 2014. Network beyond IDO in psychiatric disorders: revisiting neurodegeneration hypothesis. Prog. Neuropsychopharmacol. Biol. Psychiatry 48, 304-313.

Myint, A.M., Kim, Y.K., Verkerk, R., Scharpe, S., Steinbusch, H., Leonard, B., 2007. Kynurenine pathway in major depression: evidence of impaired neuroprotection. J. Affect Disord. 98, 143-151.

Myint, A.M., Schwarz, M.J., Verkerk, R., Mueller, H.H., Zach, J., Scharpe, S., Steinbusch, H.W., Leonard, B.E., Kim, Y.K., 2011. Reversal of imbalance between kynurenic acid and 3-hydroxykynurenine by antipsychotics in medicationnaive and medication-free schizophrenic patients. Brain Behav. Immun. 25, 1576-1581.

Nabi, R., Serajee, F.J., Chugani, D.C., Zhong, H., Huq, A.H., 2004. Association of tryptophan 2,3 dioxygenase gene polymorphism with autism. Am. J. Med. Genet. B Neuropsychiatr. Genet. 125B, 63-68.

Najjar, S., Pearlman, D.M., Alper, K., Najjar, A., Devinsky, O., 2013. Neuroinflammation and psychiatric illness. J. Neuroinflamm. 10, 43.

Nemeth, H., Robotka, H., Kis, Z., Rozsa, E., Janaky, T., Somlai, C., Marosi, M., Farkas, T., Toldi, J., Vecsei, L., 2004. Kynurenine administered together with probenecid markedly inhibits pentylenetetrazol-induced seizures. An electrophysiological and behavioural study. Neuropharmacology 47, 916-925.

Nemeth, H., Toldi, J., Vecsei, L., 2006. Kynurenines, Parkinson's disease and other neurodegenerative disorders: preclinical and clinical studies. J. Neural Transm. Suppl. 285-304.

Nikkheslat, N., Zunszain, P.A., Horowitz, M.A., Barbosa, I.G., Parker, J.A., Myint, A.M., Schwarz, M.J., Tylee, A.T., Carvalho, L.A., Pariante, C.M., 2015. Insufficient glucocorticoid signaling and elevated inflammation in coronary heart disease patients with comorbid depression. Brain Behav. Immun. 48, 8-18.

O'Connor, J.C., Lawson, M.A., Andre, C., Moreau, M., Lestage, J., Castanon, N., Kelley, K.W., Dantzer, R., 2009. Lipopolysaccharide-induced depressive-like behavior is mediated by indoleamine 2,3-dioxygenase activation in mice. Mol. Psychiatry $14,511-522$.

Ogawa, T., Matson, W.R., Beal, M.F., Myers, R.H., Bird, E.D., Milbury, P., Saso, S., 1992. Kynurenine pathway abnormalities in Parkinson's disease. Neurology 42. $1702-1706$.

Okuda, S., Nishiyama, N., Saito, H., Katsuki, H., 1996. Hydrogen peroxide-mediated neuronal cell death induced by an endogenous neurotoxin, 3hydroxykynurenine. Proc. Natl. Acad. Sci. U. S. A. 93, 12553-12558.

Okuda, S., Nishiyama, N., Saito, H., Katsuki, H., 1998. 3-Hydroxykynurenine, an endogenous oxidative stress generator, causes neuronal cell death with apoptotic features and region selectivity. J. Neurochem. 70, 299-307.

Opitz, C.A., Litzenburger, U.M., Sahm, F., Ott, M., Tritschler, I., Trump, S., Schumacher, T., Jestaedt, L., Schrenk, D., Weller, M., Jugold, M., Guillemin, G.J., Miller, C.L., Lutz, C., Radlwimmer, B., Lehmann, I., von Deimling, A., Wick, W., Platten, M., 2011. An endogenous tumour-promoting ligand of the human aryl hydrocarbon receptor. Nature 478, 197-203.

Oxenkrug, G.F., 2010. Tryptophan-kynurenine metabolism as a common mediator 
of genetic and environmental impacts in major depressive disorder: the serotonin hypothesis revisited 40 years later. Isr. J. Psychiatry Relat. Sci. 47, 56-63.

Pacher, P., Nivorozhkin, A., Szabo, C., 2006. Therapeutic effects of xanthine oxidase inhibitors: renaissance half a century after the discovery of allopurinol. Pharmacol. Rev. 58, 87-114.

Pantouris, G., Serys, M., Yuasa, H.J., Ball, H.J., Mowat, C.G., 2014. Human indoleamine 2,3-dioxygenase-2 has substrate specificity and inhibition characteristics distinct from those of indoleamine 2,3-dioxygenase-1. Amino Acids 46, 2155-2163.

Patki, G., Lau, Y.S., 2011. Melatonin protects against neurobehavioral and mitochondrial deficits in a chronic mouse model of Parkinson's disease. Pharmacol. Biochem. Behav. 99, 704-711.

Pellicciari, R., Amori, L., Costantino, G., Giordani, A., Macchiarulo, A., Mattoli, L., Pevarello, P., Speciale, C., Varasi, M., 2003. Modulation of the kynurine pathway of tryptophan metabolism in search for neuroprotective agents. Focus on kynurenine-3-hydroxylase. Adv. Exp. Med. Biol. 527, 621-628.

Perkins, M.N., Stone, T.W., 1982. An iontophoretic investigation of the actions of convulsant kynurenines and their interaction with the endogenous excitant quinolinic acid. Brain Res. 247, 184-187.

Pierozan, P., Zamoner, A., Soska, A.K., Silvestrin, R.B., Loureiro, S.O., Heimfarth, L. Mello E Souza, T., Wajner, M., Pessoa-Pureur, R., 2010. Acute intrastriatal administration of quinolinic acid provokes hyperphosphorylation of cytoskeletal intermediate filament proteins in astrocytes and neurons of rats. Exp. Neurol. 224, 188-196.

Pierozan, P., Ferreira, F., de Lima, B.O., Pessoa-Pureur, R., 2015. Quinolinic acid induces disrupts cytoskeletal homeostasis in striatal neurons. Protective role of astrocyte-neuron interaction. J. Neurosci. Res. 93, 268-284.

Pilotte, L., Larrieu, P., Stroobant, V., Colau, D., Dolusic, E., Frederick, R., De Plaen, E., Uyttenhove, C., Wouters, J., Masereel, B., Van den Eynde, B.J., 2012. Reversal of tumoral immune resistance by inhibition of tryptophan 2,3-dioxygenase. Proc. Natl. Acad. Sci. U. S. A. 109, 2497-2502.

Pisar, M., Forrest, C.M., Khalil, O.S., Mcnair, K., Vincenten, M.C., Qasem, S., Darlington, L.G., Stone, T.W., 2014. Modified neocortical and cerebellar protein expression and morphology in adult rats following prenatal inhibition of the kynurenine pathway. Brain Res. 1576, 1-17.

Powers, M.B., Asmundson, G.J., Smits, J.A., 2015. Exercise for mood and anxiety disorders: the state-of-the science. Cogn. Behav. Ther. 44, 237-239.

Prescott, C., Weeks, A.M., Staley, K.J., Partin, K.M., 2006. Kynurenic acid has a dual action on AMPA receptor responses. Neurosci. Lett. 402, 108-112.

Qian, F., Villella, J., Wallace, P.K., Mhawech-Fauceglia, P., Tario Jr., J.D., Andrews, C., Matsuzaki, J., Valmori, D., Ayyoub, M., Frederick, P.J., Beck, A., Liao, J., Cheney, R. Moysich, K., Lele, S., Shrikant, P., Old, L.J., Odunsi, K., 2009. Efficacy of levo-1methyl tryptophan and dextro-1-methyl tryptophan in reversing indoleamine-2,3-dioxygenase-mediated arrest of T-cell proliferation in human epithelial ovarian cancer. Cancer Res. 69, 5498-5504.

Radant, A.D., Bowdle, T.A., Cowley, D.S., Kharasch, E.D., Roy-Byrne, P.P., 1998. Does ketamine-mediated N-methyl-D-aspartate receptor antagonism cause schizophrenia-like oculomotor abnormalities? Neuropsychopharmacology 19, 434-444.

Raison, C.L., Broadwell, S.D., Borisov, A.S., Manatunga, A.K., Capuron, L., Woolwine, B.J., Jacobson, I.M., Nemeroff, C.B., Miller, A.H., 2005. Depressive symptoms and viral clearance in patients receiving interferon-alpha and ribavirin for hepatitis C. Brain Behav. Immun. 19, 23-27.

Raison, C.L., Dantzer, R., Kelley, K.W., Lawson, M.A., Woolwine, B.J., Vogt, G., Spivey, J.R., Saito, K., Miller, A.H., 2010. CSF concentrations of brain tryptophan and kynurenines during immune stimulation with IFN-alpha: relationship to CNS immune responses and depression. Mol. Psychiatry 15, 393-403.

Raison, C.L., Rutherford, R.E., Woolwine, B.J., Shuo, C., Schettler, P., Drake, D.F., Haroon, E., Miller, A.H., 2013. A randomized controlled trial of the tumor necrosis factor antagonist infliximab for treatment-resistant depression: the role of baseline inflammatory biomarkers. JAMA Psychiatry 70, 31-41.

Rajda, C., Majlath, Z., Pukoli, D., Vecsei, L., 2015. Kynurenines and multiple sclerosis: the dialogue between the immune system and the central nervous system. Int. J. Mol. Sci. 16, 18270-18282.

Rejdak, K., Bartosik-Psujek, H., Dobosz, B., Kocki, T., Grieb, P., Giovannoni, G. Turski, W.A., Stelmasiak, Z., 2002. Decreased level of kynurenic acid in cerebrospinal fluid of relapsing-onset multiple sclerosis patients. Neurosci. Lett. 331, 63-65.

Rejdak, K., Petzold, A., Kocki, T., Kurzepa, J., Grieb, P., Turski, W.A., Stelmasiak, Z., 2007. Astrocytic activation in relation to inflammatory markers during clinical exacerbation of relapsing-remitting multiple sclerosis. J. Neural Transm. 114, $1011-1015$.

Reus, G.Z., Jansen, K., Titus, S., Carvalho, A.F., Gabbay, V., Quevedo, J., 2015. Kynurenine pathway dysfunction in the pathophysiology and treatment of depression: evidences from animal and human studies. J. Psychiatr. Res. 68, 316-328.

Rohrig, U.F., Majjigapu, S.R., Vogel, P., Zoete, V., Michielin, O., 2015. Challenges in the discovery of indoleamine 2,3-dioxygenase 1 (IDO1) inhibitors. J. Med. Chem. http://dx.doi.org/10.1021/acs.jmedchem.5b00326.

Rossi, F., Valentina, C., Garavaglia, S., Sathyasaikumar, K.V., Schwarcz, R., Kojima, S. Okuwaki, K., Ono, S., Kajii, Y., Rizzi, M., 2010. Crystal structure-based selective targeting of the pyridoxal 5'-phosphate dependent enzyme kynurenine aminotransferase II for cognitive enhancement. J. Med. Chem. 53, 5684-5689.

Rozsa, E., Robotka, H., Vecsei, L., Toldi, J., 2008. The Janus-face kynurenic acid. J. Neural Transm. 115, 1087-1091.

Rung, J.P., Carlsson, A., Ryden Markinhuhta, K., Carlsson, M.L., 2005. (+)-MK-801 induced social withdrawal in rats; a model for negative symptoms of schizophrenia. Prog. Neuropsychopharmacol. Biol. Psychiatry 29, 827-832.

Saito, K., Crowley, J.S., Markey, S.P., Heyes, M.P., 1993. A mechanism for increased quinolinic acid formation following acute systemic immune stimulation. J. Biol. Chem. 268, 15496-15503.

Salter, M., Hazelwood, R., Pogson, C.I., Iyer, R., Madge, D.J., Jones, H.T., Cooper, B.R., Cox, R.F., Wang, C.M., Wiard, R.P., 1995. The effects of an inhibitor of tryptophan 2,3-dioxygenase and a combined inhibitor of tryptophan 2,3-dioxygenase and 5-HT reuptake in the rat. Neuropharmacology 34, 217-227.

Sauve, A.A., Wolberger, C., Schramm, V.L., Boeke, J.D., 2006. The biochemistry of sirtuins. Annu. Rev. Biochem. 75, 435-465.

Schuck, P.F., Tonin, A., da Costa Ferreira, G., Viegas, C.M., Latini, A., Duval Wannmacher, C.M., de Souza Wyse, A.T., Dutra-Filho, C.S., Wajner, M. 2007. Kynurenines impair energy metabolism in rat cerebral cortex. Cell. Mol. Neurobiol. 27, 147-160.

Schwarcz, R., Rassoulpour, A., Wu, H.Q., Medoff, D., Tamminga, C.A., Roberts, R.C., 2001. Increased cortical kynurenate content in schizophrenia. Biol. Psychiatry $50,521-530$.

Schwarcz, R., Bruno, J.P., Muchowski, P.J., Wu, H.-Q., 2012a. Kynurenines in the mammalian brain: when physiology meets pathology. Nat. Rev. Neurosci. 13, 465-477.

Schwarcz, R., Bruno, J.P., Muchowski, P.J., Wu, H.Q., 2012b. Kynurenines in the mammalian brain: when physiology meets pathology. Nat. Rev. Neurosci. 13, 465-477.

Schwieler, L., Erhardt, S., Erhardt, C., Engberg, G., 2005. Prostaglandin-mediated control of rat brain kynurenic acid synthesis-opposite actions by COX-1 and COX-2 isoforms. J. Neural Transm. (Vienna) 112, 863-872.

Schwieler, L., Larsson, M.K., Skogh, E., Kegel, M.E., Orhan, F., Abdelmoaty, S., Finn, A. Bhat, M., Samuelsson, M., Lundberg, K., Dahl, M.L., Sellgren, C., SchuppeKoistinen, I., Svensson, C., Erhardt, S., Engberg, G., 2015. Increased levels of IL-6 in the cerebrospinal fluid of patients with chronic schizophrenia-significance for activation of the kynurenine pathway. J. Psychiatry Neurosci. 40, 126-133.

Sharma, R., Mcmillan, C.R., Tenn, C.C., Niles, L.P., 2006. Physiological neuroprotection by melatonin in a 6-hydroxydopamine model of Parkinson's disease. Brain Res. 1068, 230-236.

Silva-Adaya, D., Perez-De La Cruz, V., Villeda-Hernandez, J., Carrillo-Mora, P., Gonzalez-Herrera, I.G., Garcia, E., Colin-Barenque, L., Pedraza-Chaverri, J. Santamaria, A., 2011. Protective effect of L-kynurenine and probenecid on 6 hydroxydopamine-induced striatal toxicity in rats: implications of modulating kynurenate as a protective strategy. Neurotoxicol. Teratol. 33, 303-312.

Smith, R.S., 1991. The macrophage theory of depression. Med. Hypotheses 35, 298-306.

Sominsky, L., Fuller, E.A., Bondarenko, E., Ong, L.K., Averell, L., Nalivaiko, E. Dunkley, P.R., Dickson, P.W., Hodgson, D.M., 2013. Functional programming of the autonomic nervous system by early life immune exposure: implications for anxiety. PLoS One 8, e57700.

Spitzer, C., Barnow, S., Volzke, H., Wallaschofski, H., John, U., Freyberger, H.J. Lowe, B., Grabe, H.J., 2010. Association of posttraumatic stress disorder with low-grade elevation of C-reactive protein: evidence from the general population. J. Psychiatr. Res. 44, 15-21.

St'astny, F., Hinoi, E., Ogita, K., Yoneda, Y., 1999. Ferrous iron modulates quinolinatemediated $[3 \mathrm{H}] \mathrm{MK}-801$ binding to rat brain synaptic membranes in the presence of glycine and spermidine. Neurosci. Lett. 262, 105-108.

Stark, J.L., Avitsur, R., Padgett, D.A., Campbell, K.A., Beck, F.M., Sheridan, J.F., 2001 Social stress induces glucocorticoid resistance in macrophages. Am. J. Physiol. Regul. Integr. Comp. Physiol. 280, R1799-R1805.

Stone, T.W., 1993. Neuropharmacology of quinolinic and kynurenic acids. Pharmacol. Rev, 45, 309-379.

Sundaram, G., Brew, B.J., Jones, S.P., Adams, S., Lim, C.K., Guillemin, G.J., 2014. Quinolinic acid toxicity on oligodendroglial cells: relevance for multiple sclerosis and therapeutic strategies. J. Neuroinflamm. 11, 204.

Swartz, K.J., During, M.J., Freese, A., Beal, M.F., 1990. Cerebral synthesis and release of kynurenic acid: an endogenous antagonist of excitatory amino acid receptors. J. Neurosci. 10, 2965-2973.

Szalardy, L., Zadori, D., Toldi, J., Fulop, F., Klivenyi, P., Vecsei, L., 2012. Manipulating kynurenic acid levels in the brain - on the edge between neuroprotection and cognitive dysfunction. Curr. Top. Med. Chem. 12, 1797-1806.

Tan, L., Yu, J.T., Tan, L., 2012. The kynurenine pathway in neurodegenerative diseases: mechanistic and therapeutic considerations. J. Neurol. Sci. 323, 1-8.

Tavares, R.G., Tasca, C.I., Santos, C.E., Alves, L.B., Porciuncula, L.O., Emanuelli, T., Souza, D.O., 2002. Quinolinic acid stimulates synaptosomal glutamate release and inhibits glutamate uptake into astrocytes. Neurochem. Int, 40, 621-627.

Ting, K.K., Brew, B., Guillemin, G., 2007. The involvement of astrocytes and kynurenine pathway in Alzheimer's disease. Neurotox. Res. 12, 247-262.

Vaidya, V.A., Marek, G.J., Aghajanian, G.K., Duman, R.S., 1997. 5-HT2A receptormediated regulation of brain-derived neurotrophic factor mRNA in the hippocampus and the neocortex. J. Neurosci. 17, 2785-2795.

van Erp, T.G., Saleh, P.A., Huttunen, M., Lonnqvist, J., Kaprio, J., Salonen, O., Valanne, L., Poutanen, V.P., Standertskjold-Nordenstam, C.G., Cannon, T.D. 2004. Hippocampal volumes in schizophrenic twins. Arch. Gen. Psychiatry 61, 346-353.

Varga, D., Heredi, J., Kanvasi, Z., Ruszka, M., Kis, Z., Ono, E., Iwamori, N., Iwamori, T., Takakuwa, H., Vecsei, L., Toldi, J., Gellert, L., 2015. Systemic L-Kynurenine sulfate administration disrupts object recognition memory, alters open field behavior and decreases c-Fos immunopositivity in C57BI/6 mice. Front. Behav. Neurosci. 
9, 157.

Vecsei, L., Szalardy, L., Fulop, F., Toldi, J., 2013. Kynurenines in the CNS: recent advances and new questions. Nat. Rev. Drug Discov. 12, 64-82.

Verstraelen, P., Pintelon, I., Nuydens, R., Cornelissen, F., Meert, T., Timmermans, J.P., 2014. Pharmacological characterization of cultivated neuronal networks: relevance to synaptogenesis and synaptic connectivity. Cell. Mol. Neurobiol. 34 $757-776$.

Vogelzangs, N., Beekman, A.T., de Jonge, P., Penninx, B.W., 2013. Anxiety disorders and inflammation in a large adult cohort. Transl. Psychiatry 3, e249.

Wang, J., Simonavicius, N., Wu, X., Swaminath, G., Reagan, J., Tian, H., Ling, L., 2006. Kynurenic acid as a ligand for orphan G protein-coupled receptor GPR35. J. Biol. Chem. 281, 22021-22028.

Webster, M.J., Knable, M.B., O'Grady, J., Orthmann, J., Weickert, C.S., 2002. Regional specificity of brain glucocorticoid receptor mRNA alterations in subjects with schizophrenia and mood disorders. Mol. Psychiatry 7, 985-994, 924.

Welch, A.N., Badawy, A.A., 1980. Tryptophan pyrrolase in haem regulation. Experiments with administered haematin and the relationship between the haem saturation of tryptophan pyrrolase and the activity of 5-aminolaevulinate synthase in rat liver. Biochem. J. 192, 403-410.

Wonodi, I., Stine, O.C., Sathyasaikumar, K.V., Roberts, R.C., Mitchell, B.D., Hong, L.E. Kajii, Y., Thaker, G.K., Schwarcz, R., 2011. Downregulated kynurenine 3monooxygenase gene expression and enzyme activity in schizophrenia and genetic association with schizophrenia endophenotypes. Arch. Gen. Psychiatry $68,665-674$

Wszelaki, N., Melzig, M.F., 2011. Research on an in vitro cell system for testing the neurotoxicity of kynurenine pathway metabolites. Pharmazie 66, 899-903.

Wu, W., Nicolazzo, J.A., Wen, L., Chung, R., Stankovic, R., Bao, S.S., Lim, C.K. Brew, B.J., Cullen, K.M., Guillemin, G.J., 2013. Expression of tryptophan 2,3dioxygenase and production of kynurenine pathway metabolites in triple transgenic mice and human Alzheimer's disease brain. PLoS One 8, e59749.

Xie, W., Cai, L., Yu, Y., Gao, L., Xiao, L., He, Q., Ren, Z., Liu, Y., 2014. Activation of brain indoleamine 2,3-dioxygenase contributes to epilepsy-associated depressivelike behavior in rats with chronic temporal lobe epilepsy. J. Neuroinflamm. 11, 41.

Ying, W., 2006. NAD+ and NADH in cellular functions and cell death. Front. Biosci. $11,3129-3148$.

Zadori, D., Klivenyi, P., Plangar, I., Toldi, J., Vecsei, L., 2011. Endogenous neuroprotection in chronic neurodegenerative disorders: with particular regard to the kynurenines. J. Cell. Mol. Med. 15, 701-717.

Zadori, D., Szalardy, L., Toldi, J., Fulop, F., Klivenyi, P., Vecsei, L., 2013. Some molecular mechanisms of dopaminergic and glutamatergic dysfunctioning in Parkinson's disease. J. Neural Transm. 120, 673-681.

Zhang, L., Guadarrama, L., Corona-Morales, A.A., Vega-Gonzalez, A., Rocha, L., Escobar, A., 2006. Rats subjected to extended L-tryptophan restriction during early postnatal stage exhibit anxious-depressive features and structural changes. J. Neuropathol. Exp. Neurol. 65, 562-570.

Zinger, A., Barcia, C., Herrero, M.T., Guillemin, G.J., 2011. The involvement of neuroinflammation and kynurenine pathway in Parkinson's disease. Park. Dis. 2011, 716859.

Zwilling, D., Huang, S.Y., Sathyasaikumar, K.V., Notarangelo, F.M., Guidetti, P., Wu, H.Q., Lee, J., Truong, J., Andrews-Zwilling, Y., Hsieh, E.W., Louie, J.Y., Wu, T., Scearce-Levie, K., Patrick, C., Adame, A., Giorgini, F., Moussaoui, S., Laue, G., Rassoulpour, A., Flik, G., Huang, Y., Muchowski, J.M., Masliah, E., Schwarcz, R., Muchowski, P.J., 2011. Kynurenine 3-monooxygenase inhibition in blood ameliorates neurodegeneration. Cell 145, 863-874.

1-Methyl-D-tryptophan and docetaxel in treating patients with metastatic solid tumors. http://clinicaltrials.gov/show/NCT01191216; (accessed 18.09.15.). 